\title{
Exploration of the tensor structure of the Higgs boson coupling to weak bosons in $e^{+} e^{-}$collisions
}

\author{
Gilad Amar, ${ }^{a}$ Shankha Banerjee, ${ }^{b}$ Stefan von Buddenbrock, ${ }^{c}$ Alan S. Cornell, ${ }^{a}$ \\ Tanumoy Mandal, ${ }^{b}$ Bruce Mellado $^{c}$ and Biswarup Mukhopadhyaya ${ }^{b}$ \\ ${ }^{a}$ National Institute for Theoretical Physics, School of Physics, University of the Witwatersrand, \\ Wits 2050, South Africa \\ ${ }^{b}$ Regional Centre for Accelerator-based Particle Physics, Harish-Chandra Research Institute, \\ Chhatnag Road, Jhusi, Allahabad - 211019, India \\ ${ }^{c}$ School of Physics, University of the Witwatersrand, \\ Wits 2050, South Africa \\ E-mail: gilad.amar@cern.ch, shankha@hri.res.in, \\ stefan.erich.von.buddenbrock@cern.ch, alan.cornell@wits.ac.za, \\ tanumoymandal@hri.res.in, bmellado@mail.cern.ch, biswarup@hri.res.in
}

ABSTRACT: Probing signatures of anomalous interactions of the Higgs boson with pairs of weak vector bosons is an important goal of an $e^{+} e^{-}$collider commissioned as a Higgs factory. We perform a detailed analysis of such potential of a collider operating at $250-$ $300 \mathrm{GeV}$. Mostly using higher dimensional operators in a gauge-invariant framework, we show that substantial information on anomalous couplings can be extracted from the total rates of $s$-and $t$-channel Higgs production. The most obvious kinematic distributions, based on angular dependence of matrix elements, are relatively less sensitive with moderate coefficients of anomalous couplings, unless one goes to higher centre-of-mass energies. Some important quantities to use here, apart from the total event rates, are the ratios of event rates at different energies, ratios of $s$-and $t$-channel rates at fixed energies, and under some fortunate circumstances, the correlated changes in the rates for $W$-boson pair-production. A general scheme of calculating rates with as many as four gauge-invariant operators is also outlined. At the end, we perform a likelihood analysis using phenomenological parametrization of anomalous $H W W$ interaction, and indicate their distinguishability for illustrative values of the strength of such interactions.

Keywords: Higgs Physics, Beyond Standard Model

ARXIV EPRINT: 1405.3957 


\section{Contents}

1 Introduction 1

2 Effective Lagrangian formalism $\quad 3$

3 Phenomenology at an $e^{+} e^{-}$collider $\quad 6$

3.1 Higgs production at an $e^{+} e^{-}$collider 6

3.2 A general expression for the cross-sections 8

$\begin{array}{lll}3.3 & \text { Energy dependence of } s \text { and } t \text {-channel cross-sections } & 11\end{array}$

3.4 More information from the total rates 11

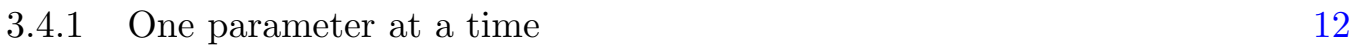

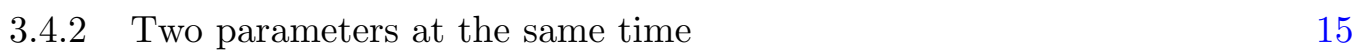

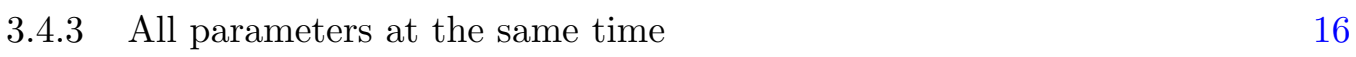

3.5 The effects on kinematic distributions 16

$\begin{array}{ll}3.6 & \text { Discussion on relevant backgrounds } \\ \end{array}$

4 Likelihood analysis for $t$-channel $\quad 22$

$\begin{array}{llr}5 & \text { Summary and conclusions } & 26\end{array}$

\section{Introduction}

Physicists are widely convinced now that they have discovered what closely resembles the Higgs boson [1, 2] postulated in the standard electroweak model (SM) [3-11]. Along with widespread exhilaration, such a development brings in questions on whether this particle carries some signature of physics beyond the standard model. Many studies in this direction have appeared [12-56] in the context of the Large Hadron Collider (LHC) where the data available so far still allow some departure from SM behaviour. Even a finite invisible branching ratio $(B R)$ for the Higgs cannot, at the moment, be ruled out [57, 58]. The issue can be probed through careful measurements of the couplings of the Higgs (or Higgs-like scalar) to various pairs of SM particles. Among them, the couplings to pairs of vector bosons $(H V V)$ are measured in a relatively more reliable manner. This possibility has been explained in the context of an $e p$ collider too [59, 60].

In view of the cumulative demand for a closer probe on the $H V V$ couplings (and of course the couplings to other SM particles), the most desirable endeavour, however, is to build an electron-positron collider which provides a clean environment for precise measurements of Higgs interaction strengths. The first step is of course to develop a Higgs factory (at $\sqrt{s} \approx 250-300 \mathrm{GeV}$ ). Such a machine will not only produce the Higgs boson copiously near resonance, but is also the first step before an $e^{+} e^{-}$machine at even higher energies 
is developed. In this paper, we incorporate some observations regarding the signatures of anomalous $H V V$ couplings, manifest through higher dimensional operators (HDOs), at a Higgs factory. Other studies performed for an $e^{+} e^{-}$machine can be found in [61].

If the couplings arise through physics at a scale higher than that of electroweak symmetry breaking, then the resulting higher-dimensional effective interactions are expected to be gauge invariant. Such interactions have not only been identified, but constraints on their coefficients have also been obtained from the LHC data [52, 62-66]. In view of such analyses, the coefficients are often restricted to such values where many cherished kinematic distributions may fail to reveal their footprints. In the current study, we point out some features which influence the detectability (or otherwise) of the higher-dimensional couplings at a Higgs factory. At the same time, we emphasise some possible measurements that can elicit their signatures even for relatively small coefficients of such operators.

We concentrate on two Higgs production channels, namely, $e^{+} e^{-} \longrightarrow Z H$ (the $s$ channel process) and $e^{+} e^{-} \longrightarrow \nu \bar{\nu} H$ (the $t$-channel process, which we separate with the help of a simple kinematic cut around the Higgs boson energy). In principle, the HDOs that will constitute our report can influence the rates in both channels. In contrast, the most obvious kinematic distributions, namely, those based on the angular dependence of matrix elements, drawn with moderate values of their coefficients do not show a perceptible difference with respect to the SM situation. Keeping this in view, we underscore the following points here:

1. The $s$-channel process has substantial rates at $\leq 300 \mathrm{GeV}$ or thereabout. We show, through an analysis of the production amplitude squared, why one cannot expect significantly different angular distributions in this channel at such energies, if one uses moderate values of the operator coefficients.

2. The $t$-channel process can have appreciable production rates at high energies $(\approx \mathrm{a}$ $\mathrm{TeV}$ ), too. Because of the production of two neutrinos in the final state, this process provides limited phase-space for the exploration of the tensor structure of the $H W W$ coupling. Here it is attempted to exploit the full kinematics of the Higgs boson by means of a correlated two-dimensional likelihood analysis.

3. We show that, given such impediment, it is possible to uncover signatures of the aforementioned BSM operators through measurements of rates at two different energies, which also cancels many systematic uncertainties. In general, the energy dependence of the rates can be sensitive to anomalous couplings.

4. The very fact that the additional operators should be electroweak gauge invariant imply not only higher-dimensional $H V V$ interactions $(V=W, Z, \gamma)$ but also anomalous $W W V$ interactions $(V=Z, \gamma)$ whose strengths are related to the former. We show that the concomitant variations in Higgs production and $\mathrm{W}$-pair production at Higgs factories may elicit the presence of such BSM interactions.

5. We also show that if the centre-of-mass energy (CME) of the colliding particles is $\approx 500 \mathrm{GeV}$ or more, then even moderate values of the operator coefficients can show some differences in the kinematic distributions. 
6. Lastly, we perform the analysis in a framework that allows one to retain all the gauge-invariant operators at the same time.

We summarise the gauge invariant couplings in the next section, and subsequently point out the 'phenomenological' anomalous couplings they lead to. In section 3, we take up the $s$ and $t$-channel Higgs production cross-sections in turn, and explain why one cannot expect too much out of kinematic distributions at Higgs factory energies, so long as the BSM coupling coefficients are subject to constraints imposed by the LHC data. Their detectable signatures through event ratios at two energies, and also via the simultaneous measurement of $W$-pair production are predicted in section 3. A likelihood analysis and some related issues, mostly in terms of the phenomenological forms to which all new couplings reduce, are found in section 4 . We summarise our conclusions in section 5 .

\section{Effective Lagrangian formalism}

In this paper, we adopt two types of effective Lagrangian parametrizations which are commonly used in the literature to probe the anomalous $H V V$ (where $V=W, Z, \gamma$ ) interactions. In one parametrization, we take the most general set of dimension- 6 gauge invariant operators which give rise to such anomalous $H V V$ interactions. In the other one, we parametrize the $H V V$ vertices with the most general Lorentz invariant structure. Although, this formalism is not the most transparent one from the viewpoint of the gauge structure of the theory, it is rather simple and more experiment-friendly. Both formalisms modify the $H V V$ vertices by introducing non-standard momentum-dependent terms.

We assume that the SM is a low-energy effective theory of a more complete perturbation theory valid below a cut-off scale $\Lambda$. In the present study, we are concerned mainly with the Higgs sector. The first order corrections to the Higgs sector will come from gauge invariant dimension 6 operators as there is only one dimension-5 operator which contributes to the neutrino masses. The relevant additional Lorentz structures in $H V V$ interactions are necessarily of dimensions higher than four. If they arise as a consequence of integrating out physics at a higher scale, all such operators will have to be invariant under $\mathrm{SU}(2)_{L} \times \mathrm{U}(1)_{Y}$. A general classification of such operators is found in the literature [67-70]. The lowest order CP-conserving operators which are relevant for Higgs phenomenology are

- The operators containing the Higgs doublet $\Phi$ and its derivatives:

$$
\mathcal{O}_{\Phi, 1}=\left(D_{\mu} \Phi\right)^{\dagger} \Phi \Phi^{\dagger}\left(D^{\mu} \Phi\right) ; \quad \mathcal{O}_{\Phi, 2}=\frac{1}{2} \partial_{\mu}\left(\Phi^{\dagger} \Phi\right) \partial^{\mu}\left(\Phi^{\dagger} \Phi\right) ; \quad \mathcal{O}_{\Phi, 3}=\frac{1}{3}\left(\Phi^{\dagger} \Phi\right)^{3}
$$

- The operators containing the Higgs doublet $\Phi$ (or its derivatives) and bosonic field strengths:

$$
\begin{aligned}
& \mathcal{O}_{G G}=\Phi^{\dagger} \Phi G_{\mu \nu}^{a} G^{a \mu \nu} ; \quad \mathcal{O}_{B W}=\Phi^{\dagger} \hat{B}_{\mu \nu} \hat{W}^{\mu \nu} \Phi ; \quad \mathcal{O}_{W W}=\Phi^{\dagger} \hat{W}_{\mu \nu} \hat{W}^{\mu \nu} \Phi \\
& \mathcal{O}_{W}=\left(D_{\mu} \Phi\right)^{\dagger} \hat{W}^{\mu \nu}\left(D_{\nu} \Phi\right) ; \quad \mathcal{O}_{B B}=\Phi^{\dagger} \hat{B}_{\mu \nu} \hat{B}^{\mu \nu} \Phi ; \quad \mathcal{O}_{B}=\left(D_{\mu} \Phi\right)^{\dagger} \hat{B}^{\mu \nu}\left(D_{\nu} \Phi\right)
\end{aligned}
$$


where $\hat{W}^{\mu \nu}=i \frac{g}{2} \sigma_{a} W^{a \mu \nu}$ and $\hat{B}^{\mu \nu}=i \frac{g^{\prime}}{2} B^{\mu \nu}$ and $g, g^{\prime}$ are respectively the $\mathrm{SU}(2)_{L}$ and $\mathrm{U}(1)_{Y}$ gauge couplings. $W_{\mu \nu}^{a}=\partial_{\mu} W_{\nu}^{a}-\partial_{\nu} W_{\mu}^{a}-g \epsilon^{a b c} W_{\mu}^{b} W_{\nu}^{c}, B_{\mu \nu}=\partial_{\mu} B_{\nu}-\partial_{\nu} B_{\mu}$ and $G_{\mu \nu}^{a}=\partial_{\mu} G_{\nu}^{a}-\partial_{\nu} G_{\mu}^{a}-g_{s} f^{a b c} G_{\mu}^{b} G_{\nu}^{c}$. The Higgs doublet is denoted by $\Phi$ and its covariant derivative is given as $D_{\mu} \Phi=\left(\partial_{\mu}+\frac{i}{2} g^{\prime} B_{\mu}+i g \frac{\sigma_{a}}{2} W_{\mu}^{a}\right) \Phi$.

Following are the properties of the aforementioned HDOs:

- $\mathcal{O}_{\Phi, 1}$ : does not preserve custodial symmetry and is therefore severely constrained by the $T$-parameter (or equivalently the $\rho$ parameter). It modifies the SM $H Z Z$ and $H W W$ couplings by unequal multiplicative factors.

- $\mathcal{O}_{\Phi, 2}$ : preserves custodial symmetry and modifies the SM $H Z Z$ and $H W W$ couplings by multiplicative factors. This operator modifies the Higgs self-interaction as well.

- $\mathcal{O}_{\Phi, 3}$ : modifies only the Higgs self-interaction.

- $\mathcal{O}_{G G}$ : introduces $H G G$ coupling which is same in structure as the SM effective $H G G$ coupling. Since our discussion is limited to the context of an $e^{+} e^{-}$collider and as we will also not consider the gluonic decay mode of the Higgs, we will not discuss this operator any further.

- $\mathcal{O}_{B W}$ : drives the tree-level $Z \leftrightarrow \gamma$ mixing and is therefore highly constrained by the electroweak precision test (EWPT) data [62].

- $\mathcal{O}_{W W}, \mathcal{O}_{W}, \mathcal{O}_{B B}, \mathcal{O}_{B}$ : modifies the $H V V$ couplings by introducing new Lorentz structure in the Lagrangian. They are not severely constrained by the EWPT data $[63,64]$.

Hence for the Higgs sector, we will choose our basis as $\mathcal{O}_{i} \in\left\{\mathcal{O}_{W W}, \mathcal{O}_{W}, \mathcal{O}_{B B}, \mathcal{O}_{B}\right\}$. In the presence of the above operators, the Lagrangian is parametrised as

$$
\mathcal{L}=\kappa\left(\frac{2 m_{W}^{2}}{v} H W_{\mu}^{+} W^{\mu-}+\frac{m_{Z}^{2}}{v} H Z_{\mu} Z^{\mu}\right)+\sum_{i} \frac{f_{i}}{\Lambda^{2}} \mathcal{O}_{i}
$$

where $\kappa$ is the scale factor of the SM-like coupling, something which needs to be accounted for when considering BSM physics. $f_{i}$ is a dimensionless coefficient which denotes the strength of the $i^{\text {th }}$ operator and $\Lambda$ is the cut-off scale above which new physics must appear. We keep $\kappa$ to be the same for the $H W W$ and $H Z Z$ couplings so that there is no unacceptable contribution to the $\rho$-parameter. Another operator considered in this work is $\mathcal{O}_{W W W}=\operatorname{Tr}\left[\hat{W}_{\mu \nu} \hat{W}^{\nu \rho} \hat{W}_{\rho}^{\mu}\right]$. This only affects the triple gauge boson couplings and does not affect the Higgs sector.

The effective Lagrangian which affects the Higgs sector is

$$
\begin{aligned}
\mathcal{L}_{\mathrm{eff}}= & g_{H W W}^{(1)}\left(W_{\mu \nu}^{+} W^{-\mu} \partial^{\nu} H+\text { h.c. }\right)+g_{H W W}^{(2)} H W_{\mu \nu}^{+} W^{-\mu \nu} \\
& +g_{H Z Z}^{(1)} Z_{\mu \nu} Z^{\mu} \partial^{\nu} H+g_{H Z Z}^{(2)} H Z_{\mu \nu} Z^{\mu \nu} \\
& +g_{H Z \gamma}^{(1)} A_{\mu \nu} Z^{\mu} \partial^{\nu} H+g_{H Z \gamma}^{(2)} H A_{\mu \nu} Z^{\mu \nu}+g_{H \gamma \gamma} H A_{\mu \nu} A^{\mu \nu},
\end{aligned}
$$


where

$$
\begin{array}{rlrl}
g_{H W W}^{(1)} & =\left(\frac{g M_{W}}{\Lambda^{2}}\right) \frac{f_{W}}{2} ; & g_{H W W}^{(2)} & =-\left(\frac{g M_{W}}{\Lambda^{2}}\right) f_{W W} \\
g_{H Z Z}^{(1)} & =\left(\frac{g M_{W}}{\Lambda^{2}}\right) \frac{c^{2} f_{W}+s^{2} f_{B}}{2 c^{2}} ; & g_{H Z Z}^{(2)} & =-\left(\frac{g M_{W}}{\Lambda^{2}}\right) \frac{s^{4} f_{B B}+c^{4} f_{W W}}{2 c^{2}} \\
g_{H Z \gamma}^{(1)} & =\left(\frac{g M_{W}}{\Lambda^{2}}\right) \frac{s\left(f_{W}-f_{B}\right)}{2 c} ; & g_{H Z \gamma}^{(2)}=\left(\frac{g M_{W}}{\Lambda^{2}}\right) \frac{s\left(s^{2} f_{B B}-c^{2} f_{W W}\right)}{c} \\
g_{H \gamma \gamma} & =-\left(\frac{g M_{W}}{\Lambda^{2}}\right) \frac{s^{2}\left(f_{B B}+f_{W W}\right)}{2} & &
\end{array}
$$

with $s(c)$ being the sine (cosine) of the Weinberg angle. The operators $\mathcal{O}_{W}, \mathcal{O}_{B}$ and $\mathcal{O}_{W W W}$ contribute to the anomalous triple gauge boson interactions. The interactions can be summarised as

$$
\mathcal{L}_{W W V}=-i g_{W W V}\left\{g_{1}^{V}\left(W_{\mu \nu}^{+} W^{-\mu} V^{\nu}-W_{\mu}^{+} V_{\nu} W^{-\mu \nu}\right)+\kappa_{V} W_{\mu}^{+} W_{\nu}^{-} V^{\mu \nu}+\frac{\lambda_{V}}{M_{W}^{2}} W_{\mu \nu}^{+} W^{-\nu \rho} V_{\rho}^{\mu}\right\},
$$

where $g_{W W \gamma}=g s, g_{W W Z}=g c, \kappa_{V}=1+\Delta \kappa_{V}$ and $g_{1}^{Z}=1+\Delta g_{1}^{Z}$ with

$$
\begin{aligned}
\Delta \kappa_{\gamma} & =\frac{M_{W}^{2}}{2 \Lambda^{2}}\left(f_{W}+f_{B}\right) ; & \lambda_{\gamma} & =\lambda_{Z}=\frac{3 g^{2} M_{W}^{2}}{2 \Lambda^{2}} f_{W W W} \\
\Delta g_{1}^{Z} & =\frac{M_{W}^{2}}{2 c^{2} \Lambda^{2}} f_{W} ; & \Delta \kappa_{Z} & =\frac{M_{W}^{2}}{2 c^{2} \Lambda^{2}}\left(c^{2} f_{W}-s^{2} f_{B}\right)
\end{aligned}
$$

The limits on these operators have been derived in many references. The most comprehensive of these are listed in references [52, 62-65]. These operators, even within their current limits, have been shown to modify the efficiencies of the various selection cuts for the relevant final states in the context of the LHC [66].

All of the aforementioned HDOs lead essentially to one effective coupling (each for $H W W$ and $H Z Z$ ), when $C P$-violation is neglected. These can be alternatively used in a phenomenological way for example, the $H(k) W_{\mu}^{+}(p) W_{\nu}^{-}(q)$ vertex can be parametrised as [71]:

$$
i \Gamma^{\mu \nu}(p, q) \epsilon_{\mu}(p) \epsilon_{\nu}^{*}(q)
$$

where deviations from the SM form of $\Gamma_{S M}^{\mu \nu}(p, q)=-g M_{W} g^{\mu \nu}$ would indicate the presence of BSM physics. These BSM deviations, including $C P$-violating ones (not considered among the gauge invariant operators), can be specified as

$$
\Gamma_{\mu \nu}^{B S M}(p, q)=\frac{g}{M_{W}}\left[\lambda\left(p \cdot q g_{\mu \nu}-p_{\nu} q_{\mu}\right)+\lambda^{\prime} \epsilon_{\mu \nu \rho \sigma} p^{\rho} q^{\sigma}\right]
$$

where $\lambda$ and $\lambda^{\prime}$ are the effective strengths for the anomalous CP-conserving and CPviolating operators respectively.

Precise identification of the non-vanishing nature of $\lambda, \lambda^{\prime}$ is a challenging task. If ever accomplished, it can tell us whether the modification in $H V V$-couplings are $C P$-conserving or $C P$-violating in nature and, if both are present, what their relative proportion is. Here 
we analyse the process $e^{+} e^{-} \rightarrow H \nu \bar{\nu}$ and see if there is any BSM physics involved by incorporating a likelihood analysis of the SM hypothesis tested against BSM hypotheses.

A few comments are in order on the two ways of parametrizing the anomalous Higgs couplings. The latter, of course, encapsulates all possible modified Lorentz invariant couplings in the lowest possible order, including both $C P$-conserving and $C P$-violating ones, in the coefficients $\lambda$ and $\lambda^{\prime}$ respectively. All of the anomalous $H W W$ and $H Z Z$ couplings listed in the gauge-invariant formulation reduce basically to one term if one confines oneself to a $C P$-conserving scenario. Thus we can say that the latter parametrization shows us a rather 'economic' way of relating the anomalous $H V V$ interactions to collider phenomenology. On the other hand, the process of relating the anomalous couplings to specific effective interactions is more transparent from the viewpoint of gauge structures when one uses the gauge invariant HDOs. It paves an easier path towards understanding the ultraviolet completion of the scenario. In addition to this, the formulation in terms of gauge-invariant operators relates the anomalous $H W W$ and $H Z Z$ interactions. One finds, in this way, a pattern in the departure of the $Z H$ and $\nu \bar{\nu} H$ final state production rates from the corresponding SM prediction. Finally, some of the gauge-invariant operators lead simultaneously to anomalous triple gauge boson interactions. There is thus an associated variation in the $Z H, \nu \bar{\nu} H$ and $W^{+} W^{-}$production rates as well as in the kinematic distributions associated with each final state. Such an association enables one to use various pieces of data to determine each new operator.

\section{Phenomenology at an $e^{+} e^{-}$collider}

In this section, we discuss various important Higgs production mechanisms through $H V V$ vertices at an $e^{+} e^{-}$collider. For the collider phenomenology, we have implemented the Lagrangians of eqs. (2.4) and (2.6) in FeynRules [72] to generate Universal FeynRules Model (UFO) [73] files suitable for interfacing with MadGraph [74]. We also use FORM [75] to compute many cross-sections analytically.

\subsection{Higgs production at an $e^{+} e^{-}$collider}

We concentrate on two main Higgs production mechanisms viz. $e^{+} e^{-} \rightarrow Z H$ and $e^{+} e^{-} \rightarrow$ $\nu \bar{\nu} H$, at an $e^{+} e^{-}$collider with energies ranging from $250 \mathrm{GeV}$ to $500 \mathrm{GeV}$. The $e^{+} e^{-} \rightarrow Z H$ channel includes only the $s$-channel processes $-e^{+} e^{-} \rightarrow Z^{*} / \gamma^{*} \rightarrow Z H$ (shown in figure 1(a)). Whereas $e^{+} e^{-} \rightarrow \nu \bar{\nu} H$ includes both the $s$-channel processes, $e^{+} e^{-} \rightarrow Z^{*} / \gamma^{*} \rightarrow$ $Z H \rightarrow \nu \bar{\nu} H$ as well as the $t$-channel process $e^{+} e^{-} \rightarrow \nu \bar{\nu} W^{*} W^{*} \rightarrow \nu \bar{\nu} H$ ( $W W$ fusion process as shown in figure $1(\mathrm{~b}))$.

The $s$ and $t$-channel processes have different kinematics and hence are affected differently by the inclusion of the HDOs. Moreover, the $t$-channel process allows us to explore the tensor structure of the $H W W$ vertex alone, free from any contamination from the $H Z Z$ and $H Z \gamma$ vertices. On the other hand, the $s$-channel process is free from any contamination due to the $H W W$ vertex. Hence, the measurement of the $s$-channel contribution will shed light on the tensorial nature of the $H Z Z$ and $H Z \gamma$ vertices. We, therefore, analyse the $s$ and $t$-channel processes separately to shed more light on the anomalous behaviour of the 


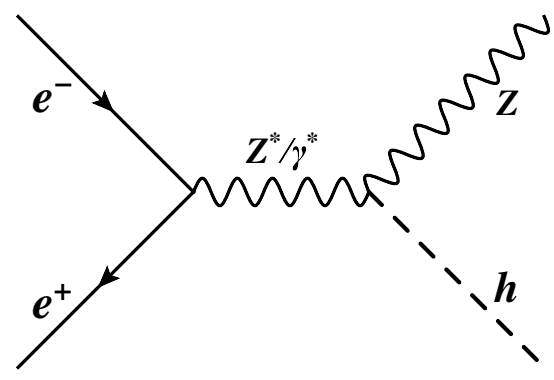

(a)

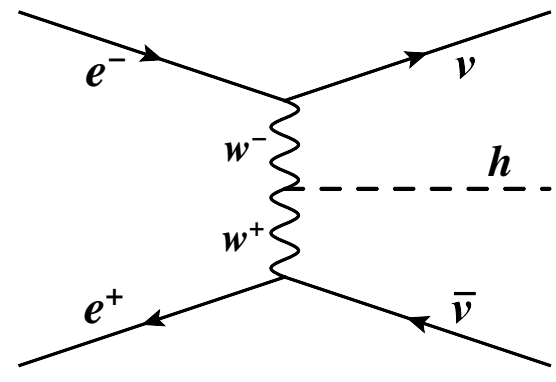

(b)

Figure 1. (a) $s$-channel Feynman diagrams (b) $t$-channel Feynman Diagram.

$H V V$ vertices. We separate the $s$-channel ( $t$-channel) contribution from the $e^{+} e^{-} \rightarrow \nu \bar{\nu} H$ events by applying a simple kinematic cut on the Higgs energy $\left(E_{H}\right)$ as follows:

$$
E_{H^{-}} \text {cut: }\left|E_{H}-\frac{s+M_{H}^{2}-M_{Z}^{2}}{2 \sqrt{s}}\right| \leq \Delta \quad\left(E_{H^{-}}^{c} \text { cut: }\left|E_{H}-\frac{s+M_{H}^{2}-M_{Z}^{2}}{2 \sqrt{s}}\right| \geq \Delta\right),
$$

where $\sqrt{s}$ is the CME of the two colliding $e^{+} e^{-}$beams and $\Delta$ is an energy-window around $E_{H}$. Here, $E_{H}^{c}$-cut is complementary to the $E_{H}$-cut. We use $\Delta=5 \mathrm{GeV}$ throughout our analysis. ${ }^{1}$ We must mention here that for the rest of this paper the $s$-channel process will be studied at the $Z H$ level without any cuts, unless otherwise specified. One can easily get an estimate of the cross-section for any decay modes of $Z$ by multiplying the appropriate BR. This is because for the $e^{+} e^{-} \rightarrow l^{+} l^{-} H$ channel, a simple invariant mass cut on the two leptons about the $Z$ boson mass will separate the $s$-channel to a very high degree. For $e^{+} e^{-} \rightarrow \nu \bar{\nu} H$, on the other hand, the cut on $E_{H}$ separates the $s$ and $t$-channels. The $s$-channel contribution surviving the cut is found to be very close to what one would have found from the rate for $l^{+} l^{-} H$, through a scaling of BRs. One is thus confident that the $E_{H^{-}}$ cut is effective in minimising mutual contamination of the $s$ and $t$-channel contributions.

It should also be mentioned here that the effects of beam energy spread are not taken into account in eq. (3.1) for simplification. While we present the basic ideas of distinguishing anomalous interactions of the Higgs, the relevant energy window for precision studies has to factor in the effects of bremsstrahlung as well as beamstrahlung (depending on whether the Higgs factory is a circular or a linear collider).

In table 1 , we show the effect of the $E_{H}$-cut on the $\nu \bar{\nu} H$ channel in the SM and in presence of HDOs for one benchmark point, BP1 $\left(\kappa=1, f_{W W}=-3, f_{W}=8, f_{B B}=\right.$ $\left.-4, f_{B}=3\right)$ which closely mimics the SM cross-section. The $E_{H^{-c u t}}$ keeps almost all the $s$ channel contribution but the $E_{H}^{c}$-cut cuts out a small portion around $E_{H}$ from the $t$-channel contribution. Therefore, the $s$-channel cross-sections after this cut increase slightly from their without-cut values due to this small $t$-channel contamination. On the other hand, the

\footnotetext{
${ }^{1}$ Typical values of $\Delta$ can be estimated from the energy uncertainties of the $b$-jets coming from the Higgs decay. The jet energy uncertainty $\Delta E_{j e t}(1 \sigma)$ of a jet having energy $E_{\text {jet }}$ are related as, $\Delta E_{\text {jet }} / E_{\text {jet }} \lesssim$ $0.3 / \sqrt{E_{j e t}}$ at the ILC [76]. For example, if there are two $b$-jets each with energy $100 \mathrm{GeV}$, the total uncertainty in their energy measurement is $\sqrt{2 \times(0.3 \times \sqrt{100})^{2}} \sim 4 \mathrm{GeV}$ (added in quadrature).
} 


\begin{tabular}{|c|c|c|c|c|c|c|c|}
\hline $\begin{array}{c}\sqrt{s} \\
(\mathrm{GeV})\end{array}$ & $\begin{array}{c}\text { Benchmark } \\
\text { point }\end{array}$ & $\begin{array}{c}\sigma_{\nu \bar{\nu} H}^{\text {tot }} \\
(\mathrm{fb})\end{array}$ & $\begin{array}{c}\sigma_{\nu \bar{\nu} H}^{s} \\
(\mathrm{fb})\end{array}$ & $\begin{array}{c}\sigma_{\nu \bar{\nu} H}^{t} \\
(\mathrm{fb})\end{array}$ & $\begin{array}{c}\sigma_{\nu \bar{\nu} H}^{\mathrm{int}} \\
(\mathrm{fb})\end{array}$ & $\begin{array}{c}\sigma_{\nu \bar{\nu} H}^{s, a c} \\
(\mathrm{fb})\end{array}$ & $\begin{array}{c}\sigma_{\nu \bar{\nu} H}^{t, a c} \\
(\mathrm{fb})\end{array}$ \\
\hline 300 & $\mathrm{SM}$ & 52.43 & 36.35 & 17.83 & -1.75 & 37.24 & 15.19 \\
& $\mathrm{BP} 1$ & 52.11 & 35.29 & 18.83 & -2.01 & 36.76 & 15.35 \\
\hline 500 & $\mathrm{SM}$ & 84.80 & 11.64 & 74.07 & -1.11 & 11.93 & 72.83 \\
& $\mathrm{BP} 1$ & 87.38 & 7.37 & 81.50 & -1.49 & 7.83 & 79.55 \\
\hline
\end{tabular}

Table 1. We show the total $\nu \bar{\nu} H$ cross-section $\left(\sigma_{\nu \bar{\nu} H}^{\text {tot }}\right)$, only $s$-channel cross-section $\left(\sigma_{\nu \bar{\nu} H}^{s}\right)$, only $t$ channel cross-section $\left(\sigma_{\nu \bar{\nu} H}^{t}\right)$ and their interference contribution $\left(\sigma_{\nu \bar{\nu} H}^{\text {int }}\right)$ for the SM $\left(\kappa=1, f_{W W}=\right.$ $\left.0, f_{W}=0, f_{B B}=0, f_{B}=0\right)$ and for HDO benchmark point BP1 $\left(\kappa=1, f_{W W}=-3, f_{W}=\right.$ $\left.8, f_{B B}=-4, f_{B}=3\right)$ for two different CMEs. We also present the $s\left(\sigma_{\nu \bar{\nu} H}^{s, a c}\right)$ and $t$-channel $\left(\sigma_{\nu \bar{\nu} H}^{t, a c}\right)$ cross-sections separated from the $\nu \bar{\nu} H$ events after applying the cut defined in eq. (3.1). The superscript ac means after cut.

$t$-channel cross-sections after cut decrease slightly from their without-cut values. We also estimate the interference between the $s$ and $t$-channel diagrams and present the numbers in table 1 . Interference contribution is expected to be tiny in the $\sqrt{s}$ region sufficiently away from the $s$-channel threshold energy $\left(M_{H}+M_{Z}\right) \approx 226 \mathrm{GeV}$. We find that the interference contribution is only $\sim 3.5 \%$ of the total cross-section for $\sqrt{s}=300 \mathrm{GeV}$, in the SM. This reaffirms the statement at the end of the previous paragraph. We also note that the inclusion of HDOs with moderate values of coefficients does not affect this contribution much. Hence, by neglecting the interference term, we approximate the total $\nu \bar{\nu} H$ cross-section as

$$
\sigma_{\nu \bar{\nu} H}^{\text {tot }} \approx \sigma_{Z H} \times B R_{Z \rightarrow \nu \bar{\nu}}+\sigma_{\nu \bar{\nu} H}^{t}
$$

where $\sigma_{Z H}$ is the $s$-channel cross section and $B R_{Z \rightarrow \nu \bar{\nu}}$ is the invisible branching fraction $(\approx 20 \%)$ of the $Z$-boson.

Figure 2 shows the invariant mass distribution of the neutrino pair for the process $e^{+} e^{-} \rightarrow \nu \bar{\nu} H$ at $\sqrt{s}=300 \mathrm{GeV}$ and for the benchmark point BP1. We separately show the distributions for the total process (which includes the $s$ and $t$ channels as well as the interference) and also the $s$ and $t$ channels separately. In an inset plot we show the distribution due to this interference. This clearly shows that it is negligible when compared to the $s$ and $t$ channel contributions. This nature generally holds for the parameter space under consideration.

\subsection{A general expression for the cross-sections}

In this analysis, we keep $\kappa, f_{W W} / \mathrm{TeV}^{2}, f_{W} / \mathrm{TeV}^{2}, f_{B B} / \mathrm{TeV}^{2}$ and $f_{B} / \mathrm{TeV}^{2}$ as free parameters. The $H W W$ vertex depends on three parameters $\left(\kappa, f_{W W}\right.$ and $\left.f_{W}\right)$ whereas the $H Z Z$ and the $H Z \gamma$ vertices depend on five parameters $\left(\kappa, f_{W W}, f_{W}, f_{B B}\right.$ and $\left.f_{B}\right)$. The $\kappa$ dependence enters the $H Z \gamma$ vertex through the $W$-loop in the effective $H Z \gamma$ vertex. The amplitude for the process $e^{+} e^{-} \rightarrow Z H / \nu \bar{\nu} H$ is a linear combination of $x_{i} \in\left\{\kappa, f_{W W}, f_{W}, f_{B B}, f_{B}\right\}$ and therefore, the cross-section can always be expressed as a bi-linear form, $\sigma\left(S, x_{i}\right)=\sum_{i, j=1}^{5} x_{i} C_{i j}(S) x_{j}$, where $C_{i j}(S)$ is the $i j^{\text {th }}$ element of the 


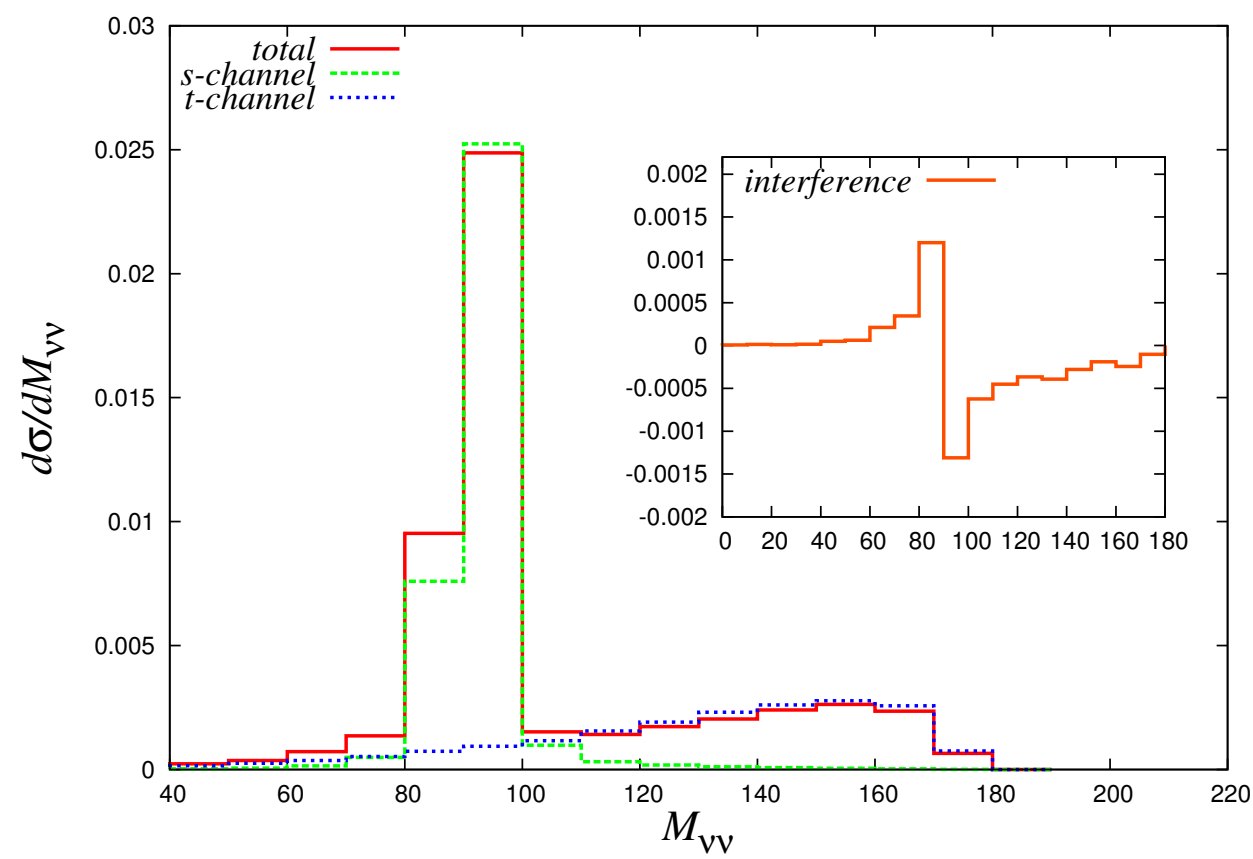

Figure 2. Invariant mass distributions of $\nu \bar{\nu}$ of the process $e^{+} e^{-} \rightarrow \nu \bar{\nu} H$ at $\sqrt{s}=300 \mathrm{GeV}$ and for the benchmark point BP1 $\left(\kappa=1, f_{W W}=-3, f_{W}=8, f_{B B}=-4, f_{B}=3\right)$. The red, green, blue histograms are for the total $(s+t+$ interference $), s$ and $t$ channels respectively. The inset (orange) plot shows the interference (total $-s-t$ ) contribution.

coefficient matrix $\mathcal{M}(\sqrt{s})$ at a CME of $\sqrt{s}$. Hence, the cross-section can be written in the following closed form

$$
\sigma(\sqrt{s})=\mathcal{X} \cdot \mathcal{M}(\sqrt{s}) \cdot \mathcal{X}^{T},
$$

where $\mathcal{X}=\left(\kappa, f_{W W}, f_{W}, f_{B B}, f_{B}\right)$ is a row vector.

The matrices of coefficients for the $e^{+} e^{-} \rightarrow Z H$ process at $\sqrt{s}=250 \mathrm{GeV}$ and $300 \mathrm{GeV}$ are

$$
\begin{aligned}
\mathcal{M}_{250}^{s, Z H}= & \left(\begin{array}{ccccc}
241.32 & -7.11 & -2.29 & -0.55 & -0.51 \\
-7.11 & 0.35 & 0.13 & -0.02 & -0.05 \\
-2.29 & 0.13 & 0.06 & -0.01 & -0.03 \\
-0.55 & -0.02 & -0.01 & 0.01 & 0.02 \\
-0.51 & -0.05 & -0.03 & 0.02 & 0.04
\end{array}\right) ; \\
\mathcal{M}_{300}^{s, Z H}= & \left(\begin{array}{ccccc}
181.67 & -6.43 & -2.99 & -0.51 & -0.71 \\
-6.43 & 0.46 & 0.18 & -0.03 & -0.08 \\
-2.99 & 0.18 & 0.14 & -0.02 & -0.06 \\
-0.51 & -0.03 & -0.02 & 0.02 & 0.03 \\
-0.71 & -0.08 & -0.06 & 0.03 & 0.08
\end{array}\right)
\end{aligned}
$$




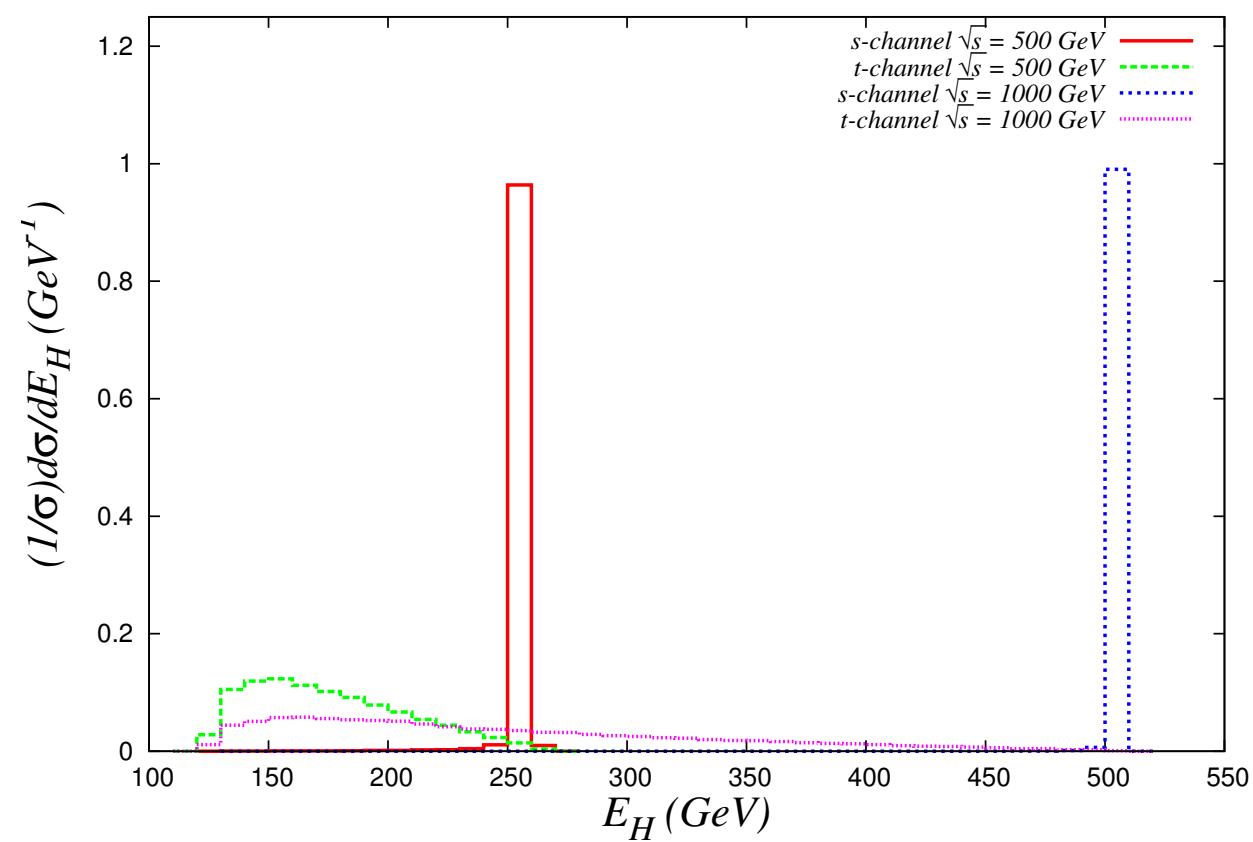

Figure 3. Normalised distributions of the Higgs energy $\left(E_{H}\right)$ for the $s$-channel (red: $\sqrt{s}=500 \mathrm{GeV}$ and blue: $\sqrt{s}=1 \mathrm{TeV}$ ) and $t$-channel (green: $\sqrt{s}=500 \mathrm{GeV}$ and magenta: $\sqrt{s}=1 \mathrm{TeV}$ ) for the benchmark point BP1.

Similar matrices for the $t$-channel process (after the $E_{H}^{c}$-cut) for the channel $e^{+} e^{-} \rightarrow$ $\nu \bar{\nu} H$ at $\sqrt{s}=250 \mathrm{GeV}$ and $300 \mathrm{GeV}$ are

$$
\begin{aligned}
& \mathcal{M}_{250}^{t, \nu \bar{\nu} H}=\left(\begin{array}{ccc}
4.63 & 5.2 \times 10^{-3} & 0.02 \\
5.2 \times 10^{-3} & 2.9 \times 10^{-4} & -1.2 \times 10^{-4} \\
0.02 & -1.2 \times 10^{-4} & 1.6 \times 10^{-4}
\end{array}\right) ; \\
& \mathcal{M}_{300}^{t, \nu \bar{\nu} H}=\left(\begin{array}{ccc}
15.36 & 0.04 & 0.07 \\
0.04 & 1.2 \times 10^{-3} & -7.7 \times 10^{-4} \\
0.07 & -7.7 \times 10^{-4} & 4.6 \times 10^{-4}
\end{array}\right)
\end{aligned}
$$

We must mention here that the matrices in eq. (3.5) are three-dimensional compared to the five-dimensional matrices in eq. (3.4) because the $t$-channel only involves the $H W W$ vertex which is not affected by the operators $\mathcal{O}_{B B}$ and $\mathcal{O}_{B}$ (eqs. (2.4), (2.5)). We also observe that in eq. (3.4), the coefficients of the matrix related to either $f_{B B}$ or $f_{B}$ are much less pronounced compared to the coefficients involving the other three parameters, viz. $\kappa, f_{W W}$ and $f_{W}$. Also from eq. (3.5) we see that barring the $(1,1)$ entry in the matrices, all the other coefficients are small implying that the HDOs will have small but non-negligible effects on the $t$-channel cross-sections for energies at the Higgs factories.

An explanation of relatively less dependence of the $t$-channel cross-section compared to the $s$-channel on the anomalous operators can also be understood from figure 3 . The plots reveal that, for the former process (essentially a vector boson fusion channel), the 
Higgs emerges with much smaller energy. The higher-dimensional couplings, on the other hand, contain derivatives which translate into a direct dependence on the energy of the Higgs, thus putting the $t$-channel process at a relative disadvantage. The Higgs energy distribution shows a longer tail for higher centre-of-mass energies, thus offering a partial recompense to the $t$-channel process for an energy as high as a TeV.

In this study we also consider the process $e^{+} e^{-} \rightarrow W^{+} W^{-}$which involves the triplegauge boson vertices $W W \gamma$ and $W W Z$. These are concomitantly affected by the operators $\mathcal{O}_{W}$ and $\mathcal{O}_{B}$. Besides, as mentioned in section 2, such vertices are also affected by the operator $\mathcal{O}_{W W W}$ which does not affect the Higgs sector. In the basis of $x_{i}^{W W} \in$ $\left\{1, f_{W}, f_{B}, f_{W W W}\right\}$, the coefficient matrix at $\sqrt{s}=300 \mathrm{GeV}$ is given by

$$
\mathcal{M}_{300}^{W W}=\left(\begin{array}{cccc}
13.48 & 1.10 \times 10^{-2} & 5.65 \times 10^{-3} & 4.24 \times 10^{-3} \\
1.10 \times 10^{-2} & 4.98 \times 10^{-4} & 5.27 \times 10^{-5} & 2.02 \times 10^{-4} \\
5.65 \times 10^{-3} & 5.27 \times 10^{-5} & 1.17 \times 10^{-4} & 1.96 \times 10^{-5} \\
4.24 \times 10^{-3} & 2.02 \times 10^{-4} & 1.96 \times 10^{-5} & 8.18 \times 10^{-4}
\end{array}\right) .
$$

As we can see above, all the $C_{i j} s$ are very small when compared to $C_{11}$, which gives us the SM cross-section. We will discuss this channel in more details later in this paper.

\subsection{Energy dependence of $s$ and $t$-channel cross-sections}

It is well-known that in SM, the cross-section for the $s$-channel falls with the CME as $1 / S$ and that for the $t$-channel, rises as $\ln S$ [77]. However, for sets of values of our parameters, different from the SM, the nature of the $s$-channel curve can be completely different from its SM-counterpart. The $t$-channel cross-section however is not affected so significantly on the introduction of HDOs as has been discussed in detail in the previous sub-section. We show the variation of the $s$ and $t$-channel processes for $\sqrt{s}$ ranging from $250 \mathrm{GeV}$ to $900 \mathrm{GeV}$. In contrast to the SM nature of a fall in the $s$-channel cross-section with energy, the introduction of HDOs does in no way ensure such a nature which can be seen in figure 4 (a) for two benchmark points (BP2 $\left(x_{i} \in\{1,0,5,0,0\}\right)$ and BP3 $\left.\left(x_{i} \in\{1,0,-5,0,0\}\right)\right)$ alongside the SM. The above two benchmark points have been chosen as the cross-sections are quite sensitive to $f_{W}$ and the two points are allowed from EWPT constraints. On the whole it is clear from the diagrams that the ratio of the $s$ and $t$-channel cross-sections in some channel at a particular energy can be an important probe to the nature of new Higgs couplings ${ }^{2}$

\subsection{More information from the total rates}

The total rates and their ratios at different CMEs can be important probes to identify the tensor structure of the $H V V$ couplings. We show how the total rates for the $s$ and $t$-channel processes are affected on the introduction of the effective operators (eqs. (3.4) and (3.5)).

\footnotetext{
${ }^{2}$ The visible rise with $\sqrt{s}$ (in figure 4(a) for the benchmark points BP2 and BP3) does not threaten unitarity, since the additional degrees of freedom responsible for the effective operators take care of it when $\sqrt{s}$ approaches $\Lambda$. The rise is not noticeable if one has the operators $\mathcal{O}_{W} / \mathcal{O}_{B B}$ instead of $\mathcal{O}_{W} / \mathcal{O}_{B}$. The different momentum dependence in the former case tames the rise with $\sqrt{s}$ as can be verified from the corresponding Feynman rules in [70].
} 


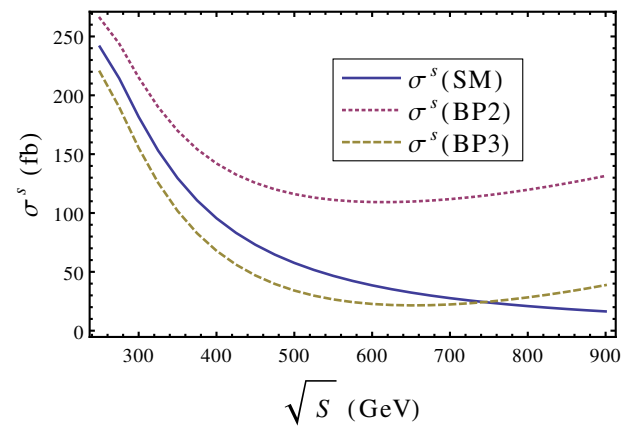

(a)

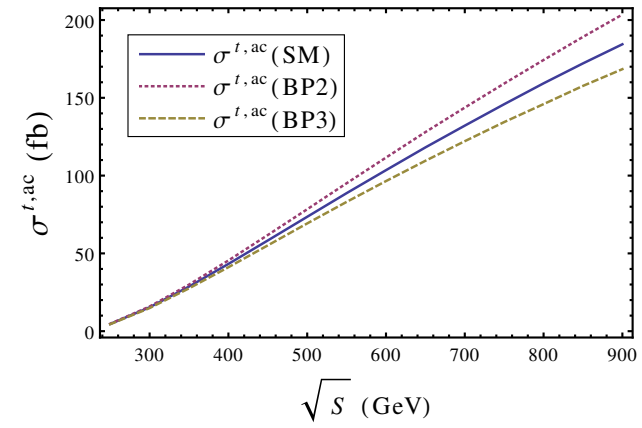

(b)

Figure 4. (a): $\sigma^{s}$ (in fb) for the channel $e^{+} e^{-} \rightarrow Z H$ and (b): $\sigma^{t, a c}$ (in fb) for the channel $e^{+} e^{-} \rightarrow$ $\nu \bar{\nu} H$ as functions of the CME, $\sqrt{s}$. The cross-sections have been computed for three benchmark points, viz. SM $\left(x_{i} \in\{1,0,0,0,0\}\right)$, BP2 $\left(x_{i} \in\{1,0,5,0,0\}\right)$ and BP3 $\left(x_{i} \in\{1,0,-5,0,0\}\right)$. The superscript ac denotes the after cut scenario.

We must make a statement about the values of the coefficients, $f_{i} / \Lambda^{2}(i$ is the index of the operator under consideration) chosen in the rest of the paper. In most cases, $f_{i} / \Lambda^{2}$ is allowed to vary in the range $[-20,20] \mathrm{TeV}^{-2}$. Now, a reasonable criterion for the validity of the effective field theory [78] is $f_{i} x(g) E^{2} / \Lambda^{2}<1$, where $x(g)$ are the $\mathrm{SU}(2)_{L} / \mathrm{U}(1)_{Y}$ factors for the operators under study and $E$ is the scale of the process. For the production case, it is the centre of mass energy of the $e^{+} e^{-}$colliding beams, which is $250-300 \mathrm{GeV}$, while for decays, it is the mass of the Higgs boson. For the production case, we perform a rough calculation taking $g \approx 0.65, g^{\prime} \approx 0.74$ and the cut-off scale $\Lambda=1 \mathrm{TeV}$. Hence, for the operator $\mathcal{O}_{W}, f_{W} x(g) E^{2} / \Lambda^{2} \approx f_{W} \frac{0.65}{2} 300^{2} / 1000^{2} \approx 0.029 f_{W}$, which can take $f_{W}$ to values $\simeq 34$. Similarly, for $\mathcal{O}_{B}$, the reach will be around $f_{B} \simeq 30$. For $\mathcal{O}_{W W}$, we have two factors of $g$ and two factors of $\frac{1}{2}$, which can take $f_{W W}$ to an even larger value. Thus the values chosen in our scan approximately conforms to the requirement of a valid effective theory.

\subsubsection{One parameter at a time}

In figures 5 and 6 , we show the variations of the $e^{+} e^{-} \rightarrow Z H$ and $e^{+} e^{-} \rightarrow \nu \bar{\nu} H$ (t-channel) cross-sections as functions of a single parameter by keeping all other parameters fixed at their SM values. We show that even for small values of the operator coefficients, the crosssections can vary significantly from the SM expectations. We also show that the ratios of the cross sections at two different energies can vary non-trivially with these parameters. If there is no new tensor structure in the $H V V$ couplings, the ratio plots will be flat horizontal curves. Any departure from a horizontal nature of such curves will shed light on new tensor structure in such $H V V$ vertices. The main sources of departure are the interference terms between the SM and HDO contributions. Such terms, occurring in both the numerator and the denominator of the ratio, carry the dependence on $f$ as well as $\sqrt{s}$.

We also remind the reader that the use of gauge invariant higher-dimensional operators implies a correlated modification in triple gauge boson couplings (eqs. (2.6), (2.7)). $f_{W}$ and $f_{B}$ are thus responsible for altering the rates of $e^{+} e^{-} \rightarrow W^{+} W^{-}$concomitantly with 


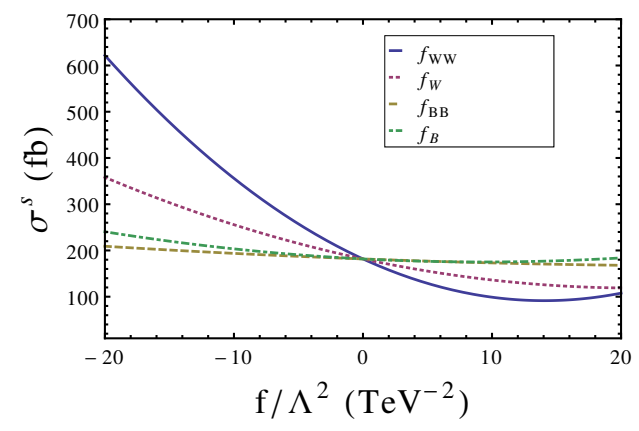

(a)

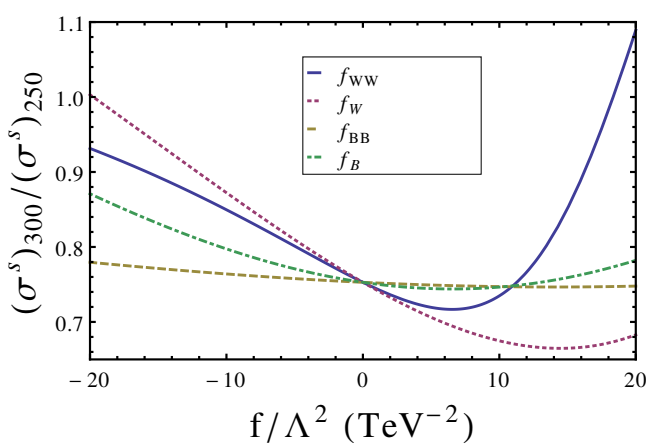

(c)

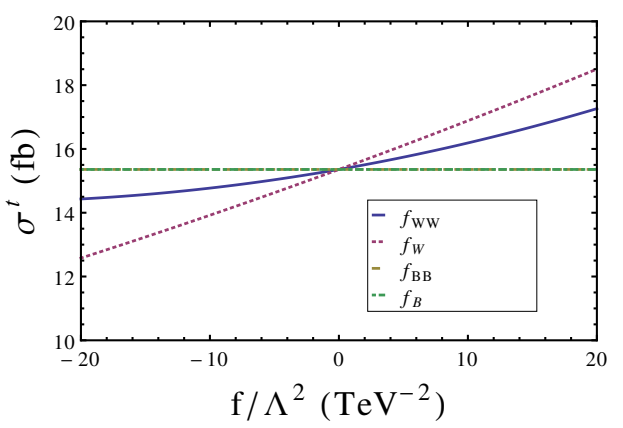

(b)

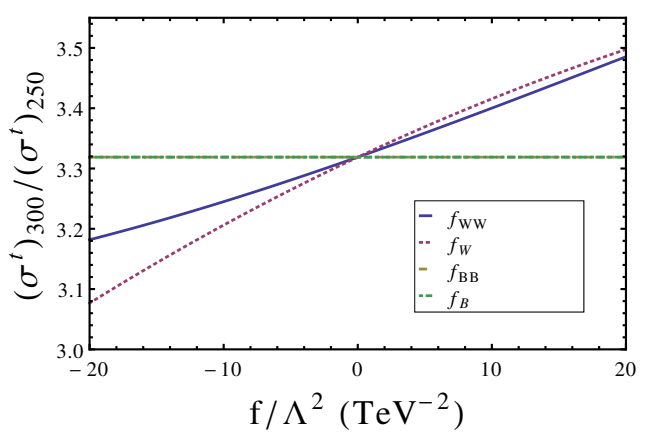

(d)

Figure 5. Variations of (a) $\sigma_{Z H}^{s}(300)(\mathrm{fb})$ and (c) $\sigma_{Z H}^{s}(300) / \sigma_{Z H}^{s}(250)$ for $e^{+} e^{-} \rightarrow Z H$ and of (b) $\sigma_{\nu \bar{\nu} H}^{t, a c}(300)(\mathrm{fb})$ and (d) $\sigma_{\nu \bar{\nu} H}^{t}(300) / \sigma_{\nu \bar{\nu} H}^{t, a c}(250)$ for $e^{+} e^{-} \rightarrow \nu \bar{\nu} H$ with $f_{W W}, f_{W}, f_{B B}, f_{B} . \kappa=1$ for all the cases. The superscript $a c$ denotes the cut in eq. (3.1). The numbers in the brackets are the CMEs.

those for Higgs boson production. Such a concomitance, if verified in an $e^{+} e^{-}$collision experiment, should point rather unmistakably at one or the other of the gauge invariant operators mentioned here. We show the modified rates of the $W W$ final state in figure 7 where we also show the effects of the operator driven by $f_{W W W}$ (which does not affect the Higgs couplings).

It should however be mentioned that the actual presence of anomalous couplings in $e^{+} e^{-} \rightarrow W^{+} W^{-}$is best reflected in a detailed study of various kinematic regions [79]. Such a study, however is not the subject of the present paper.

The main conclusion emerging from figures 5, 6 and 7 are as follows:

- In figures 5(a) and 6(a), for the process $e^{+} e^{-} \rightarrow Z H$, we find that the operator $\mathcal{O}_{W W}$ changes the cross section from its SM expectation by $\sim 30 \%$ even in the range $-5<$ $f_{W W}<5$. The major contribution to the cross section modification comes from the operators $\mathcal{O}_{W W}$ and $\mathcal{O}_{W} \cdot \mathcal{O}_{B}$ and $\mathcal{O}_{B B}$ have lesser contributions to the cross section.

- In figures 5(b) and 6(b), for the cut-applied t-channel contribution in the process $e^{+} e^{-} \rightarrow \nu \bar{\nu} H$, the operator $\mathcal{O}_{W}$ maximally affects the cross-section. The effect of $\mathcal{O}_{W W}$ is comparatively less pronounced. $\mathcal{O}_{B B}$ and $\mathcal{O}_{B}$ does not change this cross- 


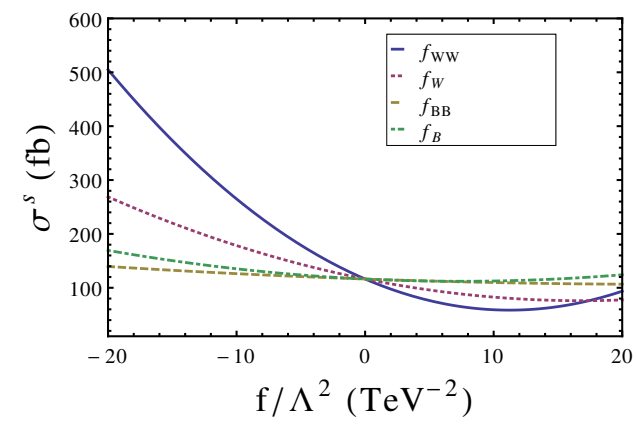

(a)

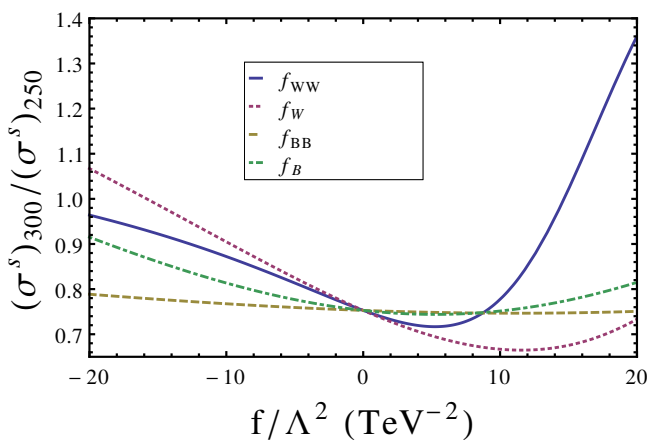

(c)

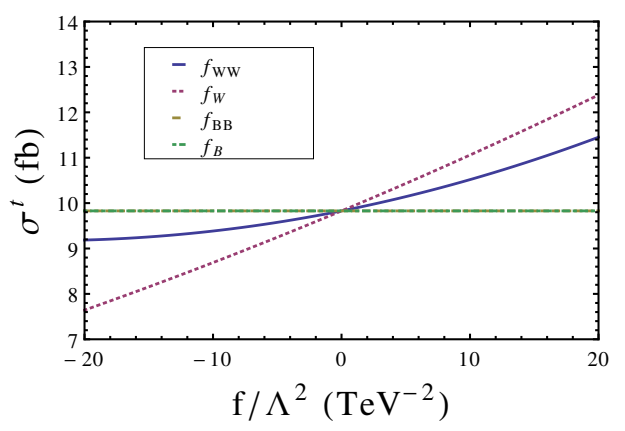

(b)

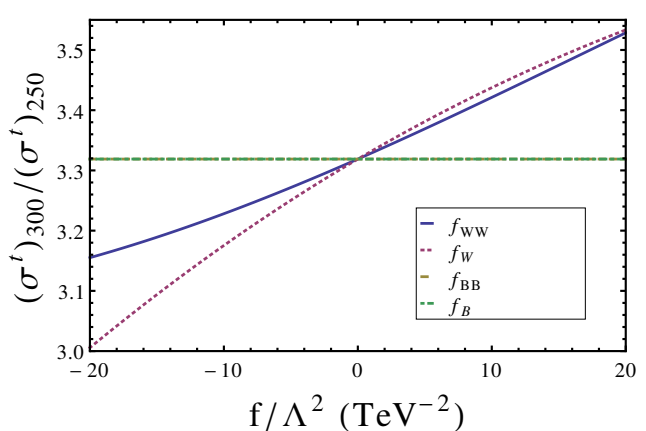

(d)

Figure 6. Variations of (a) $\sigma_{Z H}^{s}(300)$ (fb) and (c) $\sigma_{Z H}^{s}(300) / \sigma_{Z H}^{s}(250)$ for $e^{+} e^{-} \rightarrow Z H$ and of (b) $\sigma_{\nu \bar{\nu} H}^{t, a c}(300)(\mathrm{fb})$ and (d) $\sigma_{\nu \bar{\nu} H}^{t}(300) / \sigma_{\nu \bar{\nu} H}^{t, a c}(250)$ for $e^{+} e^{-} \rightarrow \nu \bar{\nu} H$ with $f_{W W}, f_{W}, f_{B B}, f_{B} . \kappa=0.8$ for all the cases. The superscript $a c$ denotes the cut in eq. (3.1). The numbers in the brackets are the CMEs.

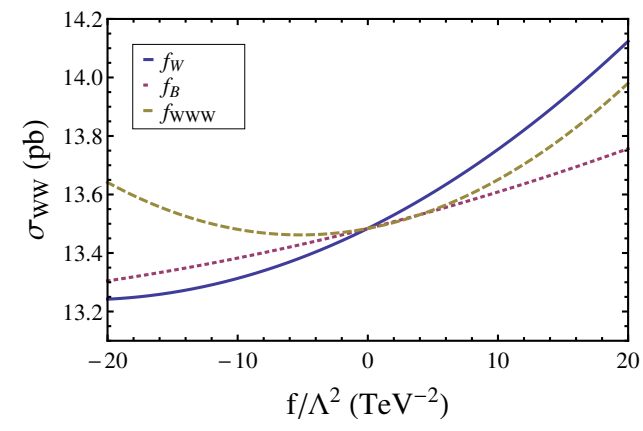

(a)

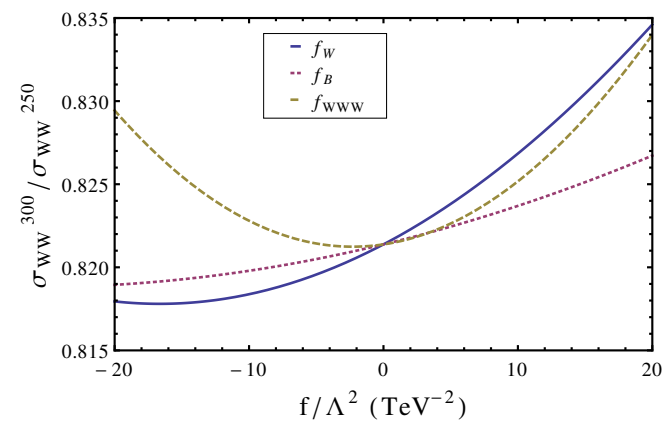

(b)

Figure 7. (a) Cross section ( $\sigma$ (in pb)) for the process $e^{+} e^{-} \rightarrow W^{+} W^{-}$for $\sqrt{s}=300 \mathrm{GeV}$ and (b) ratio of cross sections $\left(\sigma_{300} / \sigma_{250}\right)$ for the same process as functions of $f$ 's. 
section as the $H W W$ vertex is unaffected by these operators. Most importantly, it should be noted that the effect of these operators on the $t$-channel process is much less pronounced than its $s$-channel counterpart (eqs. (3.4), (3.5)).

- In figures 5(c) and 6(c), the ratio of the cross sections for the $e^{+} e^{-} \rightarrow Z H$ channel at $\sqrt{s}=300 \mathrm{GeV}$ and $\sqrt{s}=250 \mathrm{GeV}$ shows a different nature. In the range $-20<f_{i}<20$ for the four operators discussed above, the ratio changes by $\sim 33 \%$ for $\mathcal{O}_{W}$. The effect of $\mathcal{O}_{W W}$ is less than this. The change in the ratio is the least for $\mathcal{O}_{B B}$.

- In figures $5(\mathrm{~d})$ and $6(\mathrm{~d})$, the ratio of cross-sections for the cut-applied $t$-channel process varies in the range $\sim[3.1,3.5]$ for $-20<f_{i}<20$.

- We see that in figure 7 , the cross-sections do not vary significantly with the operator coefficients. This is because the $e^{+} e^{-} \rightarrow W^{+} W^{-}$channel has a strong $\nu_{e}$ mediated $t$-channel contribution which does not involve the triple-gauge boson vertex. This has a significant interference with the $s$-channel. In order to bring out the feature of the triple gauge boson vertices, we need to devise some strategy which will tame down the $t$-channel effect, such as using right-polarised electrons if one uses a linear collider.

\subsubsection{Two parameters at the same time}

In figures 8 and 9 , we show some fixed cross-section contours in the planes of two parameters varied at the same time. In figures 8 and 9, all the parameters apart from the ones shown in the axes, are kept fixed. In each of these figures, we have marked regions in brown where the cross-section is $\sigma(S M) \pm 10 \% \times \sigma(S M)$. Hence, we see that for each of these plots, some regions even with large values of the parameters can closely mimic the SM cross-section. The above statement for the ranges of the coefficients of the HDOs will be somewhat modified if we consider the Higgs decays. This is because then we will have branching ratios depending on the effects of the HDOs. Even for fermionic decays of the Higgs, which are independent of the operators under study, the $B R$ will have non-trivial effects on the operator couplings through the total decay width. But, we must mention here that unless we go to very high values of the operator coefficients, the total decay width remains close to the SM expectation and hence fermionic decay channels would show similar features as these plots. Of course, when we study the effects of all the operators in the basis that we have considered by considering every possible decay mode of the Higgs, then the higherdimensional operators will come to play at the $H V V$ decay vertices also. Hence, we will get modified bounds on the operator coefficients from a similar approach. We should mention that these operators are also constrained by the electroweak precision observables, viz. $S$, $T$ and $U$ parameters. An important observation which is carried forward from figure 5 (a) is that the $H Z Z$ and $H \gamma Z$ vertices are very less affected by the operators $\mathcal{O}_{B B}$ and $\mathcal{O}_{B}$. This fact is corroborated in figure 8 (e). The above mentioned pair of operators thus allow a wide region of parameter space which has cross-sections within $10 \%$ of the SM value.

Some salient features of figures 8 and 9 are:

- Figure 8 shows the variation of the total rate for the channel $e^{+} e^{-} \rightarrow Z H$ as functions of two parameters taken together. All the other parameters are fixed for these plots. 
In figures 8(a)-(d), the cross-section varies significantly from the SM value for the allowed ranges of the parameters. However, figure $8(\mathrm{e})$ shows a large region of the parameter space to have cross-sections similar to the SM (within 10\%).

- Figure 9 shows the variation of the cross-sections for the $t$-channel process in $e^{+} e^{-} \rightarrow$ $\nu \bar{\nu} H$ as functions of two parameters varied at the same time. Figures 9(c) and 9(d) shows a substantial amount of parameter space agreeing with the SM cross-section.

\subsubsection{All parameters at the same time}

The most general case will be to vary all the parameters simultaneously to obtain the most realistic parameter space. Here, we demonstrate this scenario for the cut-applied $t$-channel cross section in the $e^{+} e^{-} \rightarrow \nu \bar{\nu} H$ channel. In figures 10 (a), (b) and (c) we present three slices of the 3-dimensional hyper-surface. For each of these plots, there is a third parameter which has been varied. We see that a very large parameter space is allowed which can mimic the SM cross section within its $10 \%$ value. Of course these plots are for illustrative purposes only. In figure $10(\mathrm{~d})$, we have shown one such slice of the five-dimensional hyper-surface in the space of $\left(\kappa, f_{W W}, f_{W}, f_{B B}\right.$ and $\left.f_{B}\right)$ for the $s$-channel process.

Discussion on EWPT constraints. All the benchmark points chosen throughout this paper are consistent with all constraints available till date $[62,63]$. However, if one looks at the contour plots in figures 8,9 and 10, there may exist certain points which are disfavoured by the precision constraints.

\subsection{The effects on kinematic distributions}

The presence of anomalous $H V V$ vertex can in principle also affect the shapes of various kinematic distributions. In figures 11(a) and 11(b) [figures 11(c) and (d)], we show the normalised angular (angle of Higgs with the $z$-axis) distributions for the $s$-channel ( $t$-channel) processes for $\sqrt{s}=300 \mathrm{GeV}$ and $500 \mathrm{GeV}$ respectively. We find that the angular dependence for the $s$-channel is very sensitive in some regions of the parameter space allowed by the EWPT constraints and the LHC data. We also find the $\cos \theta$ dependence can be completely opposite as we increase the CME. This can be seen in figures 11(a) and 11(b), if we compare the curves for BP1. In contrast, the $t$-channel is not significantly affected by the inclusion of HDOs. The angular dependence of the differential cross-sections can be expressed as

$$
\frac{d \sigma\left(\sqrt{s}, x_{i}\right)}{d \cos \theta}=a\left(\sqrt{s}, x_{i}\right)+b\left(\sqrt{s}, x_{i}\right) \cos ^{2} \theta
$$

It is found that, between coefficients $a$ and $b$ above, $a$ is more affected by the anomalous couplings rather than $b$, unless $\sqrt{s}$ is $500 \mathrm{GeV}$ or well above that. As a result, angular distributions are insensitive to the new interactions at the proposed energy scale of a Higgs factory.

In figures 11(e) and 11(f), we show the normalised $d \sigma / d p_{T, h}$ and $d \sigma / d y_{h}$ distributions respectively for the $t$-channel where $p_{T, h}$ is the transverse momentum of the Higgs and $y_{h}$ is its rapidity. We want to emphasise that it is very difficult to see any significant 


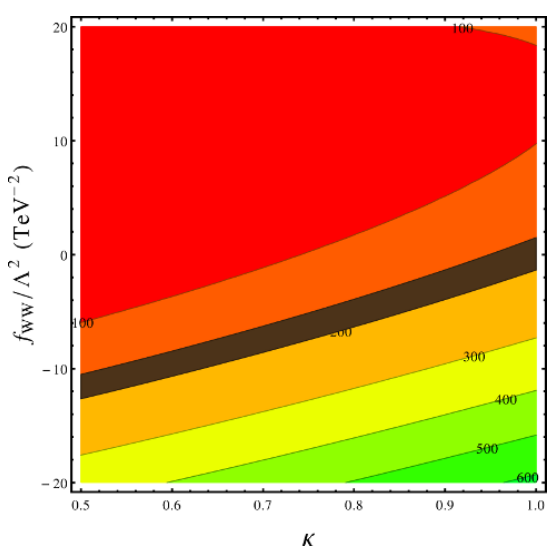

(a)

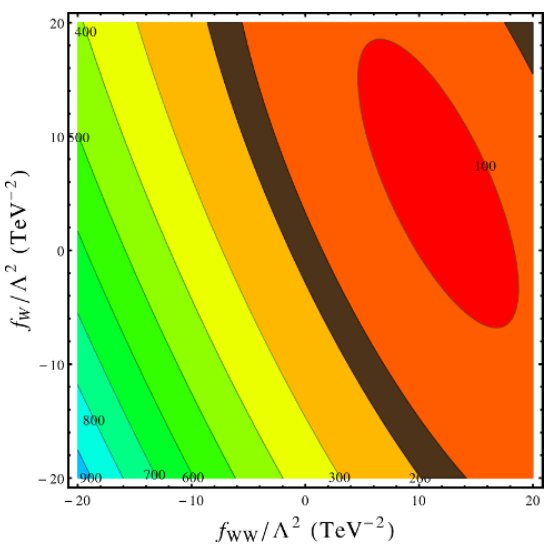

(c)

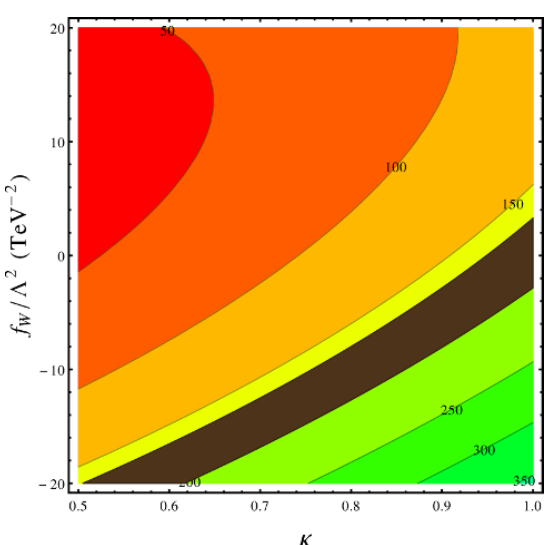

(b)

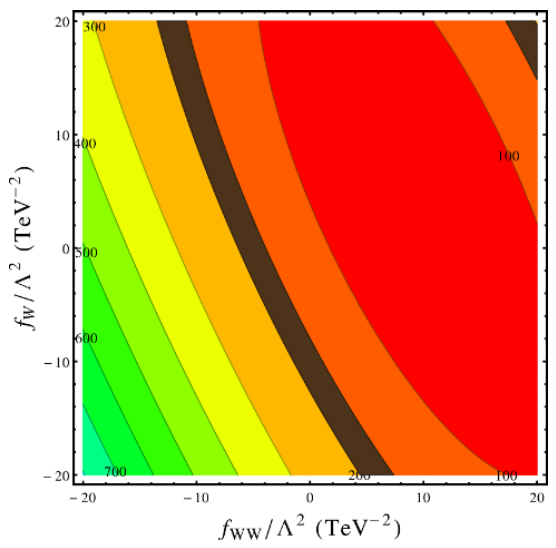

(d)

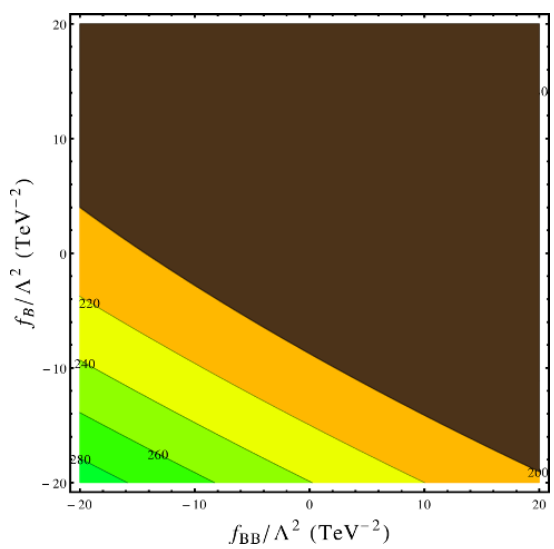

(e)

Figure 8. Variations of $\sigma_{s}^{300}$ for $e^{+} e^{-} \rightarrow Z h$ with (a) $\kappa$ and $f_{W W}$, (b) $\kappa$ and $f_{W}$, (c) $f_{W W}$ and $f_{W}$ for $\kappa=1$, (d) $f_{W W}$ and $f_{W}$ for $\kappa=0.8$ and (e) $f_{B B}$ and $f_{B}$ for $\kappa=1$. For each case all the other $f_{\mathrm{s}}$ are set to zeroes. Brown patches signify cross-sections within $\pm 10 \%$ of the SM expectation. 


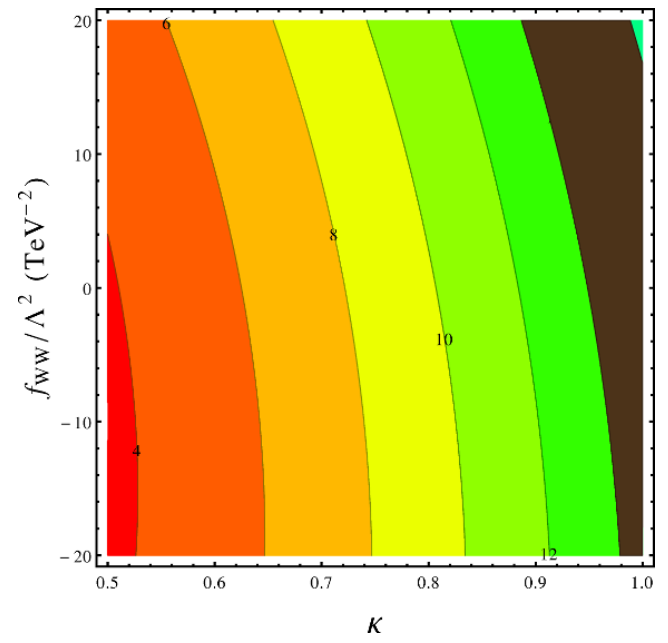

(a)

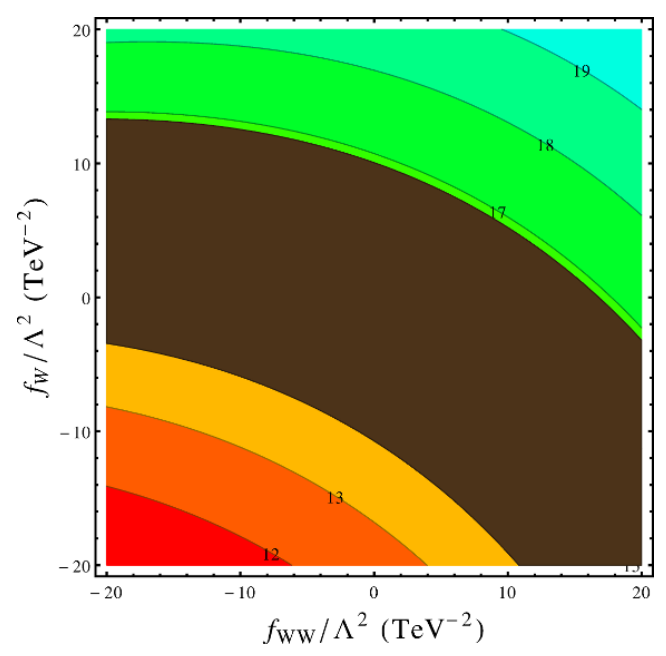

(c)

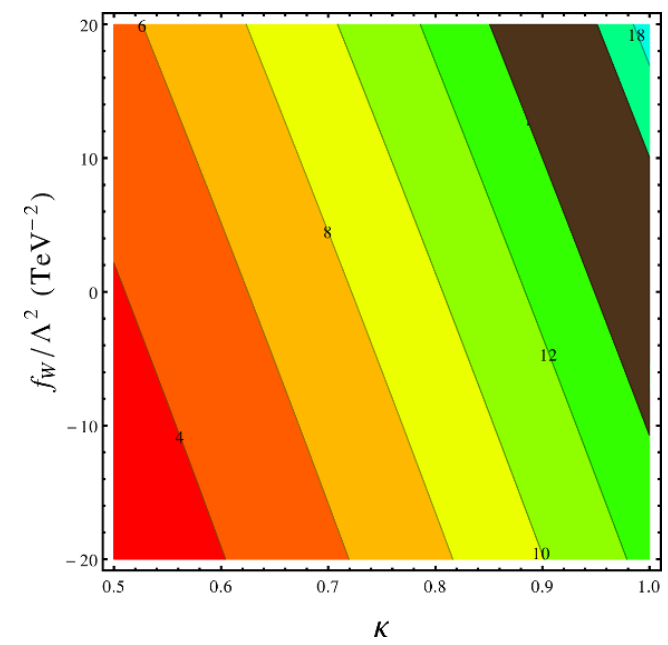

(b)

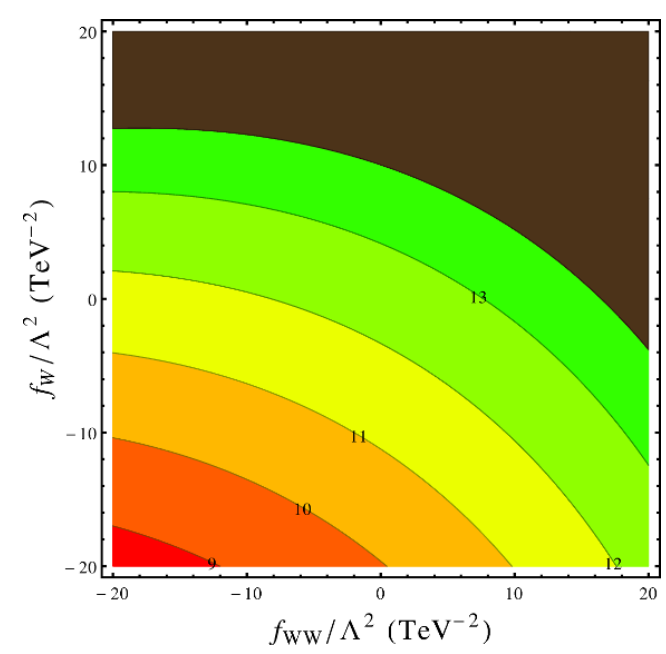

(d)

Figure 9. Variations of $\sigma_{t}^{300, a c}$ for $e^{+} e^{-} \rightarrow Z h$ with (a) $\kappa$ and $f_{W W}$, (b) $\kappa$ and $f_{W}$, (c) $f_{W W}$ and $f_{W}$ for $\kappa=1$, (d) $f_{W W}$ and $f_{W}$ for $\kappa=0.9$. For each case all the other $f_{\mathrm{S}}$ are set to zeroes. Brown patches signify cross-sections within $\pm 10 \%$ of the SM expectation.

differences in the various kinematic distributions in most of the parameter space allowed by the LHC and EWPT constraints while performing experiments with smaller CME. In both the channels, we do not consider the final decay products of the Higgs. If we consider the Higgs boson decaying to fermionic final states, then the HDOs under consideration will not affect these decay vertices and the above normalised distributions will remain intact. However, if we consider the bosonic decay modes of the Higgs, then the HDOs will affect these distributions non-trivially.

We end this subsection with the following admission. Various kinematical distributions are canonically emphasized as the best places to find the signature of non-standard Lorentz structures in interaction terms. While this expectation is not completely belied in the 


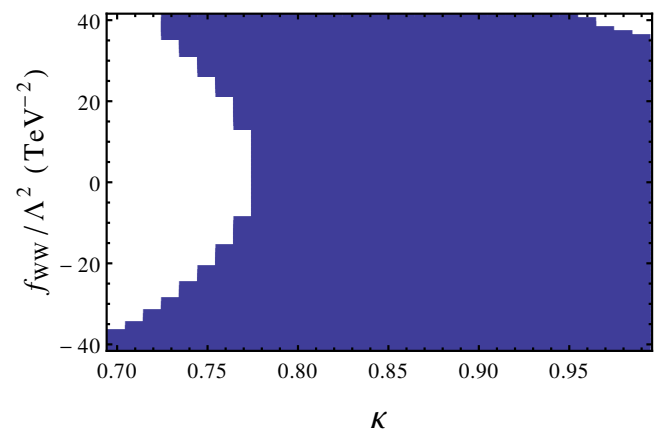

(a)

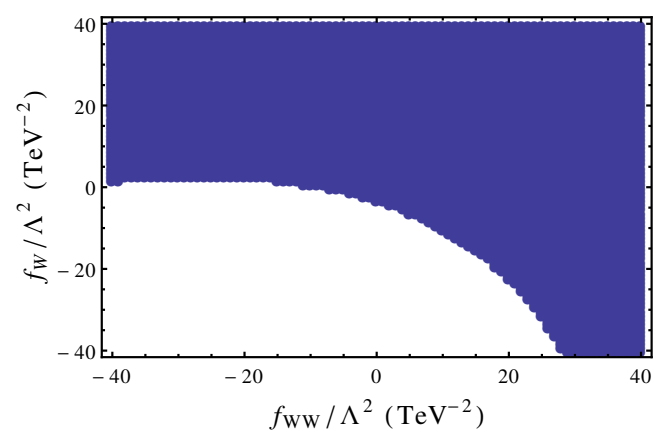

(c)

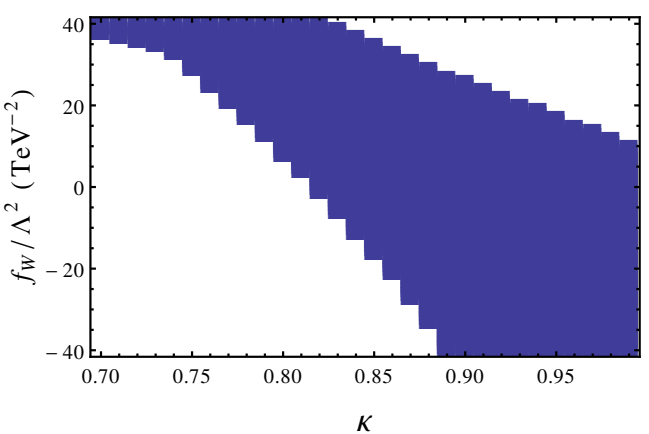

(b)

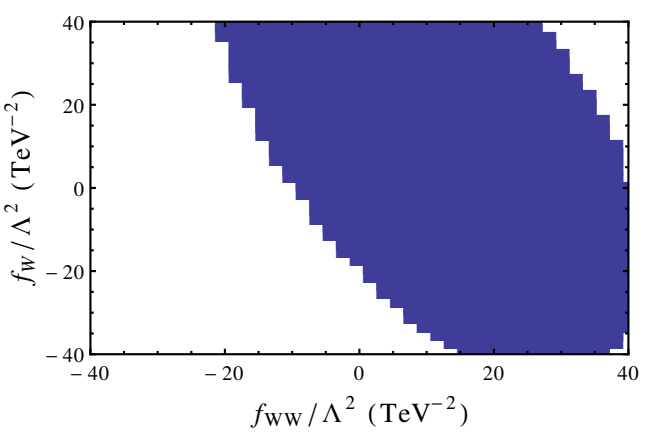

(d)

Figure 10. Allowed parameter space for $\sigma_{\nu \bar{\nu} H}^{t, a c}$ within $10 \%$ of its SM value: (a) $f_{W W}$ vs $\kappa\left(f_{W}\right.$ varied), (b) $f_{W}$ vs $\kappa$ ( $f_{W W}$ varied), (c) $f_{W}$ vs $f_{W W}$ ( $\kappa$ varied) and for $\sigma_{Z H}^{s}$ within $10 \%$ of its SM value: (d) $f_{W}$ vs $f_{W W}\left(\kappa, f_{B B}\right.$ and $f_{B}$ varied). $\sqrt{s}=300 \mathrm{GeV}$.

present case as well, we note that the anomalous couplings are reflected in distributions at relatively high CMEs. The reason behind this has already been explained above. While this prospect is encouraging, electron-positron colliders, especially those designed as Higgs factories, are likely to start operating at energies as low as $250-300 \mathrm{GeV}$. Our observation is that the imprint of anomalous couplings can be found even at such low energies at the level of total rates and their ratios. A detailed study involving all possible decay products and their various correlations can in principle go further in revealing traces of anomalous couplings. We will take up such a study in a subsequent work.

\subsection{Discussion on relevant backgrounds}

We wish to see the effects of anomalous $H V V$ couplings on the Higgs production alone. Therefore, we do not look at bosonic decay modes of Higgs and limit our discussion only to those signal processes where $H$ decays maximally to a $b \bar{b}$ pair. For the $e^{+} e^{-} \rightarrow Z H$ process, the $Z$ can either decay visibly to $b \bar{b}, j j, \ell^{+} \ell^{-}$(here $j=g, u, d, c, s$ and $\ell=e, \mu$ ) modes or invisibly to a $\nu \bar{\nu}$ pair. So the dominant backgrounds relevant for these final states are the non-Higgs $e^{+} e^{-} \rightarrow b \bar{b} b \bar{b}, b \bar{b} j j, b \bar{b} \ell^{+} \ell^{-}, b \bar{b}+\not E$. The non-Higgs $e^{+} e^{-} \rightarrow b \bar{b}+\not{E}$ process can also act as the dominant background for the $e^{+} e^{-} \rightarrow \nu \bar{\nu} H$ channel. We select events after the following kinematic cuts: trigger cuts: $p_{T}(b, j)>20 \mathrm{GeV}, p_{T}(\ell)>10 \mathrm{GeV}$, $|y(b, j)|<5.0,|y(\ell)|<2.5, \Delta R(b b, b j, j j, b \ell, j \ell)>0.4, \Delta R(\ell \ell)>0.2$. 


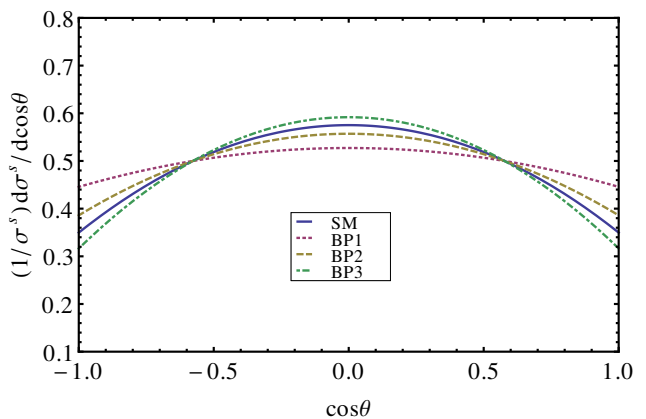

(a)

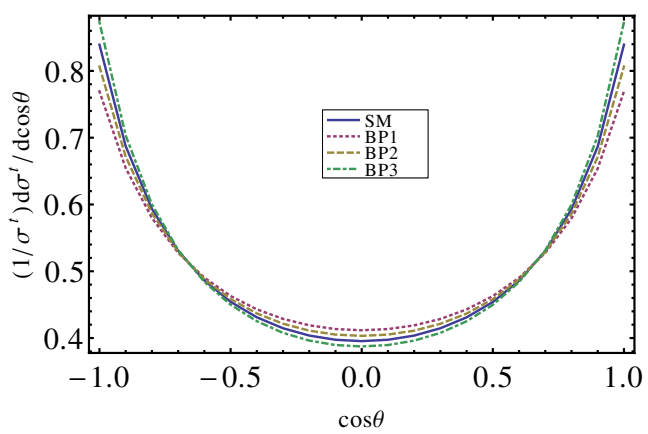

(c)

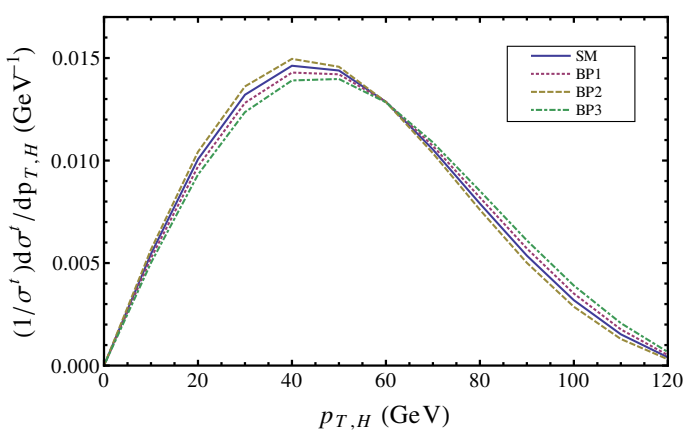

(e)

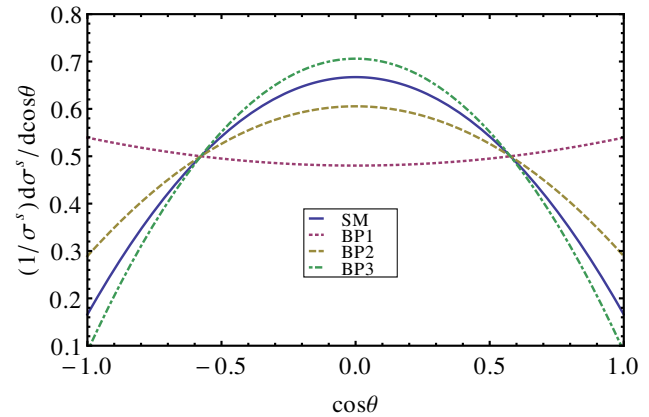

(b)

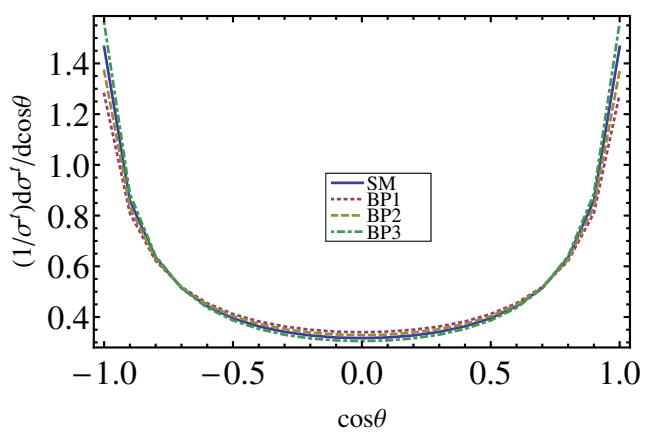

(d)

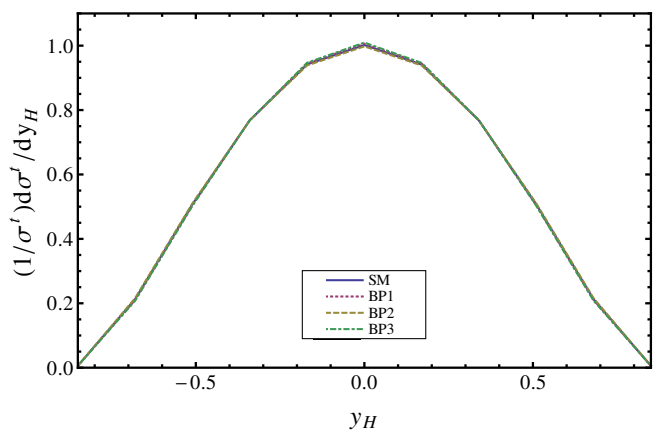

(f)

Figure 11. Normalised kinematic distributions $\left(1 / \sigma^{s}\right) d \sigma^{s} / d \cos \theta$ for the channel $e^{+} e^{-} \rightarrow Z H$ for (a) $\sqrt{s}=300 \mathrm{GeV}$ and (b) $\sqrt{s}=500 \mathrm{GeV}$. Normalised kinematic distributions $\left(1 / \sigma^{t}\right) d \sigma^{t} / d \cos \theta$ for the $t$-channel process in $e^{+} e^{-} \rightarrow \nu \bar{\nu} H$ for (c) $\sqrt{s}=300 \mathrm{GeV}$ and (d) $\sqrt{s}=500 \mathrm{GeV}$. Distributions for (e) $\left(1 / \sigma^{t}\right) d \sigma^{t} / d p_{T, H}$ and (f) $\left(1 / \sigma^{t}\right) d \sigma^{t} / d y_{H}$ for the $t$-channel process in $e^{+} e^{-} \rightarrow \nu \bar{\nu} H$ at $\sqrt{s}=$ $300 \mathrm{GeV}$. Benchmark points, viz. SM $\left(x_{i} \in\{1,0,0,0,0\}\right), \mathrm{BP} 1\left(x_{i} \in\{1,-3,8,-4,3\}\right), \operatorname{BP} 2\left(x_{i} \in\right.$ $\{1,0,5,0,0\})$ and BP3 $\left(x_{i} \in\{1,0,-5,0,0\}\right)$. 


\begin{tabular}{|c|c|c|c|c|c|c|c|}
\hline Final states & $\begin{array}{c}\sqrt{s} \\
(\mathrm{GeV})\end{array}$ & $\begin{array}{c}\sigma_{S M, t c}^{\text {sig }} \\
(\mathrm{fb})\end{array}$ & $\begin{array}{c}\sigma_{S M, a c}^{\text {sig }} \\
(\mathrm{fb})\end{array}$ & $\begin{array}{c}\sigma_{B P 1, t c}^{\text {sig }} \\
(\mathrm{fb})\end{array}$ & $\begin{array}{c}\sigma_{B P 1, a c}^{\text {sig }} \\
(\mathrm{fb})\end{array}$ & $\begin{array}{c}\sigma_{t c}^{b k g} \\
(\mathrm{fb})\end{array}$ & $\begin{array}{c}\sigma_{a c}^{b k g} \\
(\mathrm{fb})\end{array}$ \\
\hline$b \bar{b} l^{+} l^{-}$ & 250 & 2.68 & 2.46 & 2.76 & 2.52 & 10.33 & 0.09 \\
& 300 & 2.33 & 1.91 & 2.31 & 1.83 & 9.17 & 0.07 \\
\hline$b \bar{b}+E$ & 250 & 12.25 & 10.31 & 12.36 & 10.53 & 20.53 & 0.33 \\
& 300 & 13.67 & 9.79 & 13.26 & 9.62 & 18.00 & 0.29 \\
\hline
\end{tabular}

Table 2. We show the signal and backgrounds for two different final states, viz. $b \bar{b} l^{+} l^{-}$and $b \bar{b}+\not \subset$. $\sigma_{t c}$ 's are the cross-sections after the basic trigger cuts mentioned above and $\sigma_{a c}$ 's are the crosssections after the channel-specific cuts. The analysis has been done for the SM and the benchmark point BP1 $\left(x_{i} \in\{1,-3,8,-4,3\}\right)$.

Finally we estimate two of the aforementioned backgrounds by applying the cuts below:

- Non-Higgs $e^{+} e^{-} \rightarrow b b \ell \ell$

We demand the two $b$ 's to fall within the Higgs-mass window and the two $\ell$ 's to fall within the $Z$-mass window as follows:

$$
\left|M(b b)-M_{h}\right|<10 \mathrm{GeV} \text { AND }\left|M(\ell \ell)-M_{Z}\right|<10 \mathrm{GeV}
$$

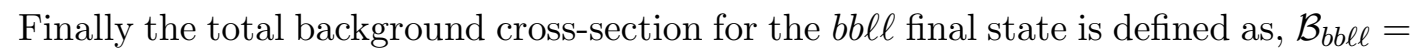
$\eta_{b}^{2} \sigma_{b b \ell \ell}$ where $\eta_{b}$ is the $b$-tagging efficiency which we take as 0.6 for our analysis. The signal is also scaled by the same factor, $\eta_{b}^{2}$.

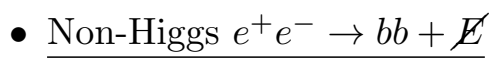

We demand the two $b$ 's to fall within the Higgs-mass window, $\left|M(b b)-M_{h}\right|<10 \mathrm{GeV}$. Here the background is $\mathcal{B}_{b b+E}=\eta_{b}^{2} \sigma_{b b+E^{*}}$. The signal ${ }^{3}$ has also been scaled by the $b$-tagging efficiency.

Alongside the issue of distinctness of the presence of the anomalous couplings, it is of interest to find out about the reach of a Higgs factory, or to know down to what strength the anomalous couplings can be detected. This information can be found in figure 12 . There we have have plotted the quantities $\mathcal{S}=\left|\sigma_{B S M}^{H}-\sigma_{S M}^{H}\right|$ and $\mathcal{B}=\sigma_{S M}^{H}+\sigma_{S M}^{N H}$ for computing the significance. Here, $H(N H)$ signifies sub-processes which involve (does not involve) the Higgs.

In table 2, we show the cross-sections for both the signal and background scenarios. For the signal we have considered two benchmark points, viz. SM and BP1 $\left(x_{i} \in\right.$ $\{1,-3,8,-4,3\}))$. We show the cross-sections once after applying just the trigger cuts (designated with the subscript $t c$ ) and next by applying the channel-specific selection cuts (written with a subscript $a c$ ) along with the basic trigger cuts. All the numbers have been multiplied by $\eta_{b}^{2}$. We see that the effects of the invariant mass selection cuts on the signal

\footnotetext{
${ }^{3}$ The channel $e^{+} e^{-} \rightarrow H+E \rightarrow b \bar{b}+E$ also includes diagrams involving the triple-gauge boson vertices. These effects are almost nullified when the selection cuts for this channel are employed.
} 


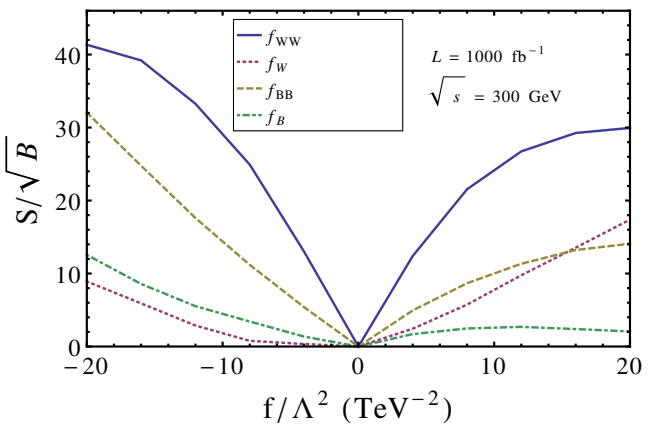

(a)

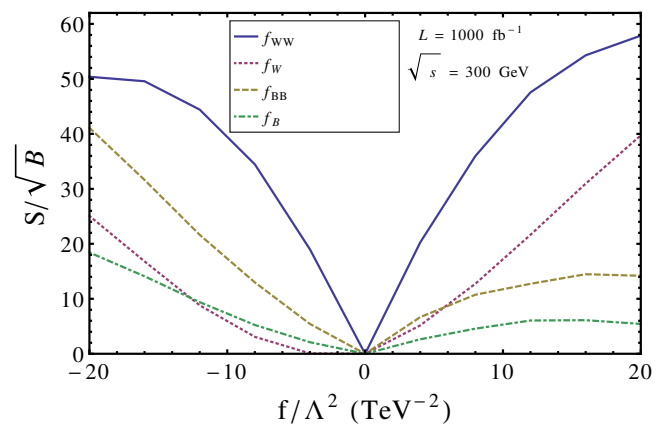

(b)

Figure 12. Significance $(\mathcal{S} / \sqrt{\mathcal{B}})$ as functions of $f_{i} / \Lambda^{2}$ for $\kappa=1$ at $\sqrt{s}=300 \mathrm{GeV}$ for (a) $e^{+} e^{-} \rightarrow$ $b b \ell \ell$ and $(\mathrm{b}) e^{+} e^{-} \rightarrow b b+E$.

cross-sections are negligible whereas these are very effective in reducing the backgrounds almost completely.

The study performed here is at parton level. Shower, hadronization and detector effects are expected to have an impact on the effective cross-sections reported in table 2. That said, these effects will not change the conclusions of the paper.

\section{Likelihood analysis for $t$-channel}

The kinematics of the final state associated to the $s$-channel production has been studied extensively in the past. As pointed out in section 1, the $t$-channel production provides limited phase-space because the momenta of the outgoing neutrinos cannot be disentangled experimentally. This leaves the Higgs boson kinematics as the only handle to explore the nature of the $H W W$ coupling. Studies are documented in the literature with the use of the Higgs boson momentum as a means to gain sensitivity. Here we attempt to fully exploit the kinematics of the Higgs boson by means of a correlated two-dimensional likelihood analysis. The primary intent of this section is to shed light on the relative improvement of this two-dimensional approach, rather than determining absolute sensitivity to the size of anomalous couplings. The latter requires a detailed study that carefully incorporates experimental effects. This is beyond the scope of this paper.

We use a test-statistic (TS) to distinguish the BSM hypothesis from its SM counterpart by defining the logarithm of a profile likelihood ratio $\left(q_{i j}=\ln \lambda_{i j}\right)$ for two different hypotheses $i$ and $j$ defined as

$$
q_{i j}=\ln \lambda_{i j}=\ln \frac{L\left(P_{i} \mid D_{i}\right)}{L\left(P_{j} \mid D_{i}\right)}
$$

where $\lambda_{i j}$ is the ratio of two likelihood functions $L\left(P_{i} \mid D_{i}\right)$ and $L\left(P_{j} \mid D_{i}\right)$ describing two different hypotheses, ${ }^{4} D_{i}$ is the data set used and $P_{i, j}$ are the probability density functions.

\footnotetext{
${ }^{4}$ Alternatively, its reciprocal is also sometimes used, depending on the analysis required. It should be noted here that both likelihoods are constructed using the same $D_{i}$, but different $P_{i}$ s.
} 


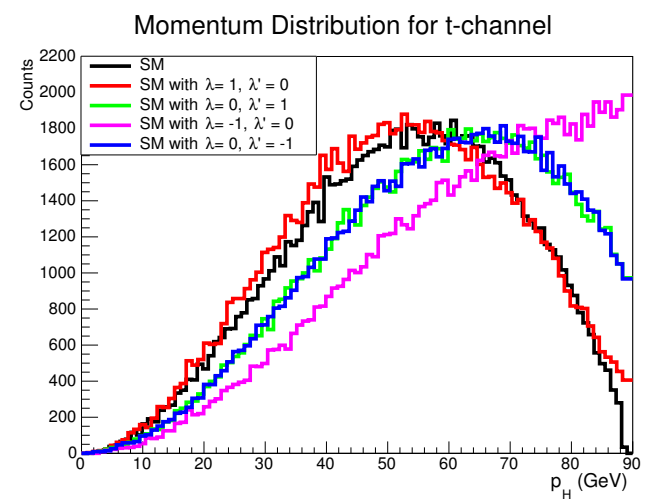

(a)

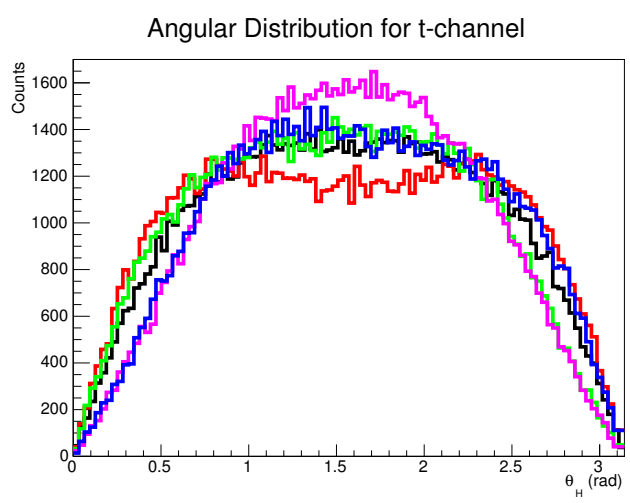

(b)

Figure 13. Normalised kinematic distributions of (a) Higgs momentum, $p_{H}$ and (b) the angle of the Higgs with the beam-axis, $\theta_{H}$ for different benchmark points for the $t$-channel process at $\sqrt{s}=250 \mathrm{GeV}$.

Due to the discrete nature of the probabilities in this analysis, the likelihood functions are defined as products of binned Poisson probabilities over all channels and bins [1]. From the TS, a $p$-value can be calculated to quantify the extent to which a hypothesis can be rejected. In general, a $p$-value is a portion of the area under a normalised TS which, after calculation, is the percentage confidence level (CL) by which a hypothesis can be rejected.

In Monte Carlo (MC) studies, these TSs emerge as binned peaks which show up on running pseudo-experiments, each of which returns a value for the TS based on a randomly generated set of pseudo-data. The number of pseudo-data points generated is fixed by the cross-section of the process being studied. The TSs concerned in this analysis are always produced in pairs, in order to discriminate between the SM and BSM hypotheses. This pair of TSs is represented as

$$
q_{U}=\ln \frac{L\left(P_{S M} \mid D_{S M}\right)}{L\left(P_{B S M} \mid D_{S M}\right)} \quad \text { and } \quad q_{L}=\ln \frac{L\left(P_{S M} \mid D_{B S M}\right)}{L\left(P_{B S M} \mid D_{B S M}\right)} .
$$

The $q_{U}$ TS tends to have a more positive value due to its ordering, and we refer to it as the upper TS for our purposes, while we refer to $q_{L}$ as the lower TS. A hypothesis can be rejected by calculating the associated $p$-value as follows

$$
p=\int_{m_{q_{U}}}^{\infty} q_{L}(q) d q,
$$

where $m_{q_{U}}$ is the median of the upper TS, $q_{U}$. The confidence by which a hypothesis can be rejected, can alternatively be quantified by knowing the significance of the separation between the two TSs. The median-significance, $Z_{\text {med }}$, is defined as the number of standard deviations between the median of $q_{L}$ and the left edge of the $p$-value area, that is, the median of $q_{U}$.

As stated above, we focus on the $t$-channel process (in $e^{+} e^{-} \rightarrow \nu \bar{\nu} H$ ) which has not been studied as extensively as the $s$-channel. The $s$-channel ( $t$-channel) contributions can 


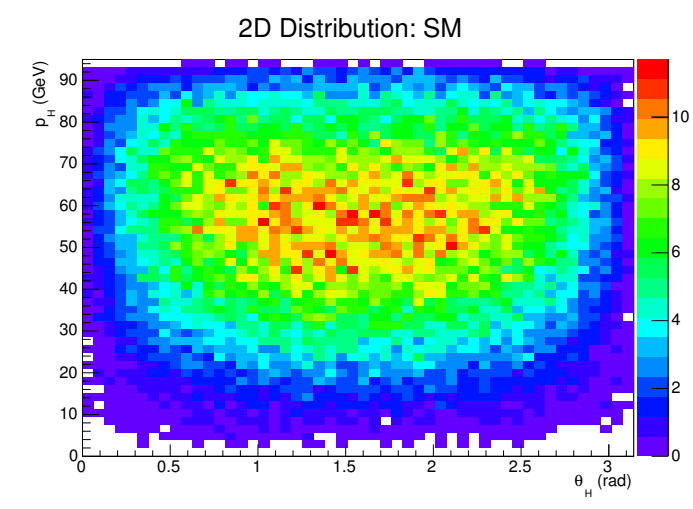

(a)

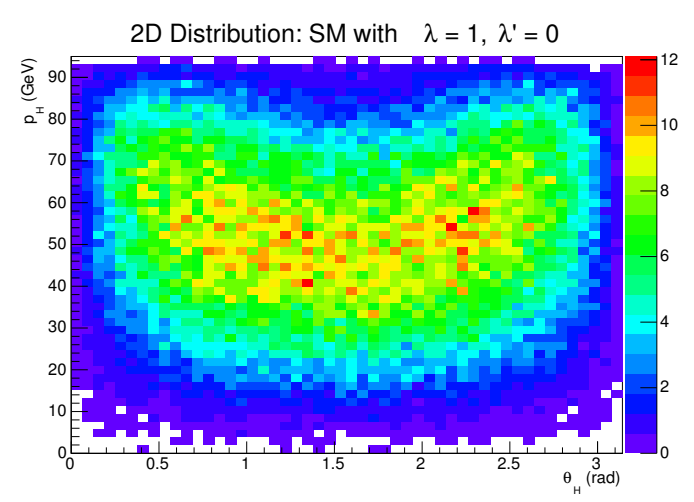

(b)

Figure 14. Two dimensional histograms showing the correlation of the $t$-channel Higgs momentum, $p_{H}$ and the angle of the Higgs with the beam-axis, $\theta_{H}$ at $\sqrt{s}=250 \mathrm{GeV}$. The $z$-axis is an indication of the frequency of events, in arbitrary units. The effect of the correlation can be seen by noting how the BSM parameter $\lambda$ affects the distribution.

be separated out from the $\nu \bar{\nu} H$ events by applying the $E_{H}$-cut $\left(E_{H}^{c}\right.$-cut) in eq. (3.1). For this purpose, we work with the phenomenological parametrization of anomalous $H W W$ interaction characterised by $\lambda$ and $\lambda^{\prime}$, as defined in eq. (2.9).

In our analysis, the vertices for the Lagrangians in the SM and in BSM with spin0 bosons are calculated in FEynRules [72] and passed to the event-generator MADGRAPH [74], which is used for the generation of the matrix elements for Higgs production in the $t$ - and $s$-channels. MC samples are produced at parton level. Effects related to detector resolution are taken into account when defining requirements to suppress the contamination from the $s$-channel process (see eq. (3.1)).

We set the stage for the likelihood analysis by showing some plots for distributions in terms of $\lambda$ and $\lambda^{\prime}$. In figures 13(a) and (b), we show the $p_{H}$ (Higgs momentum) and $\theta_{H}$ (the angle of the Higgs with the beam-axis) distributions respectively for the $t$-channel at $\sqrt{s}=250 \mathrm{GeV}$. We see that significant deviations from the SM can be seen. This is in contrast to what was shown for the gauge invariant formulation (in figure 11) because there we stick to moderate values of the parameter coefficients, whereas for example, here, $\left.\left\{\lambda=1, \lambda^{\prime}=0\right\} \Rightarrow x_{i} \approx\{1,77,0,0,0\}\right)$. In figures $14(\mathrm{a})$ and (b), two dimensional histograms in $p_{H^{-}} \theta_{H}$ plane are shown for the SM and a BSM (SM with $\lambda=1, \lambda^{\prime}=0$ ) benchmark point respectively at $\sqrt{s}=250 \mathrm{GeV}$.

A likelihood analysis for each BSM hypothesis is performed for integrated luminosities of $1 \mathrm{fb}^{-1}, 5 \mathrm{fb}^{-1}$ and $10 \mathrm{fb}^{-1}$. The number of pseudo-data points in each analysis is determined from the SM cross section. The $Z_{\text {med }}$ for the $1 \mathrm{fb}^{-1}$ case are plotted as functions of the CME for each hypothesis as shown in figure 15. These plots show the power of using two dimensional distributions in likelihood analysis. The likelihood analysis is performed using a total number of 100,000 pseudo-experiments for each TS. The two dimensional distributions, examples of which are shown in figure 14, are also included in the likelihood analysis to demonstrate the effect of the correlation between the two variables, $p_{H}$ and $\theta_{H}$. 


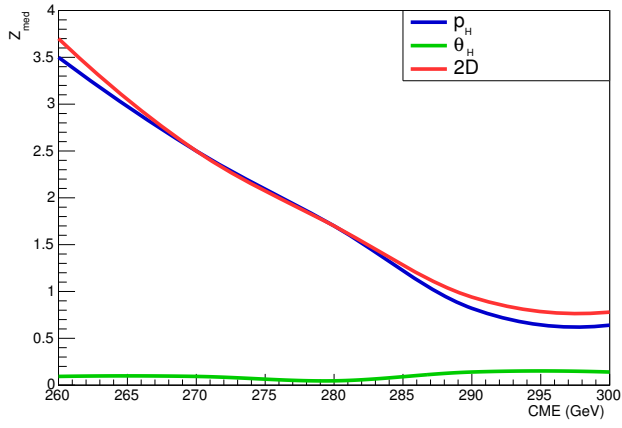

(a)

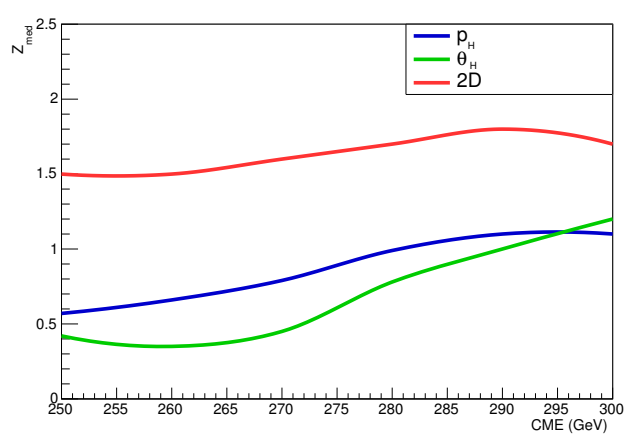

(c)

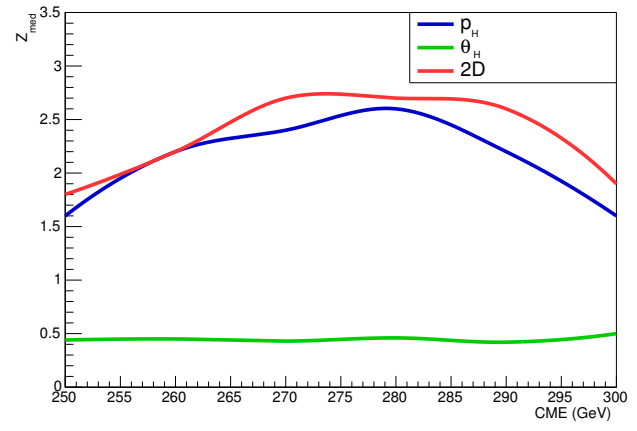

(b)

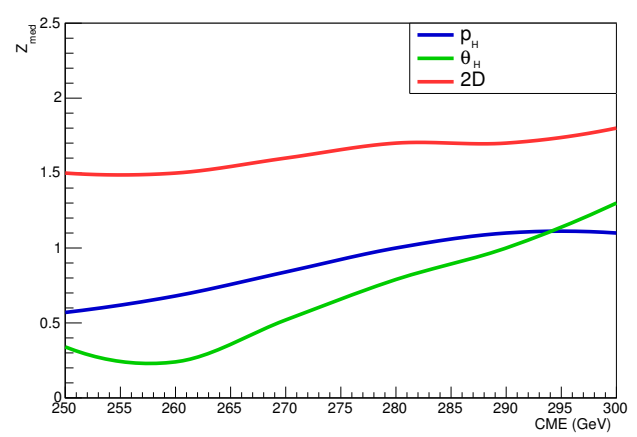

(d)

Figure 15. Median significance values for likelihood analyses done with both one dimensional and two dimensional distributions. (a) SM with $\lambda=1$, (b) SM with $\lambda=-1$, (c) SM with $\lambda^{\prime}=1$ and (d) SM with $\lambda^{\prime}=-1$. Results are obtained with $1 \mathrm{fb}^{-1}$ of integrated luminosity.

Figure 15 displays the significance for one-dimensional analyses using the Higgs boson momentum and the polar angle separately. Results are shown for illustration purposes for $1 \mathrm{fb}^{-1}$ of integrated luminosity. Conclusions drawn here are found not to depend on the integrated luminosity in the range studied here. The corresponding results for the combined 2D likelihood are shown. The upper two plots correspond to admixtures with the CP-even term. The sensitivity of the polar angle is significantly less than that of the Higgs boson momentum. The lower plots display the corresponding results for admixtures with the CP-odd term. In this case the sensitivity of the polar angle is similar to that of the momentum. As a result, the improvement from the $2 \mathrm{D}$ analysis is significant, to the extent that the sensitivity can be enhanced by about a factor of two. The sensitivity of the angular variable grows with the CME.

The results provide a good motivation for the role of an electron positron collider in understanding the nature of the $H V V$ couplings. The plots in figure 15 show the utility in using two dimensional distributions in discerning the rejection of hypotheses. That is, using the same accrued data from two separate one dimensional distributions, one can enhance the confidence in rejecting hypotheses. The correlation of the two dimensional distributions thus carries vital information about the dynamics of the processes which are studied in $e^{+} e^{-}$collisions. 


\section{Summary and conclusions}

We have attempted to demonstrate the efficacy as well as limitations of an $e^{+} e^{-}$Higgs factory operating at $250-300 \mathrm{GeV}$ in probing anomalous, higher-dimensional couplings of a Higgs to $W$-and $Z$-pairs, suppressed by a scale $\mathcal{O}(\mathrm{TeV})$. For this purpose, we have mostly adhered to the set of gauge-invariant operators that can lead to such interactions, since it is such terms that are expected to emerge on integrating out physics above the electroweak symmetry breaking scale. We have utilised the consequent correlation of the anomalous $H W W, H Z Z$ and $H Z \gamma$ couplings, and also the concomitant effect on $Z W W / \gamma W W$ interactions, as reflected in gauge boson pair-production rates.

The general conclusion reached by this study is that the total rates can be quite useful as probes of higher-dimensional operators. Based on this, we have performed a detailed analysis of the cross-sections for $s$-and $t$-channel Higgs production, specifying event selection criteria for minimising their mutual contamination. A general scheme of computing the rates with more than one gauge-invariant operators has been outlined. Based on such an analysis, we conclude that, even with the additional operators well within the erstwhile experimental bounds (including those form the LHC), a number of observations can probe them at a Higgs factory. These include not only the individual total cross-sections but also their ratios at different values of $\sqrt{s}$ and also the ratio of the $s$-and the $t$-channel Higgs production rates at fixed energies. We also indicate the correlated variation of $W$ pair production rates. The Higgs production rate contours with more than one type of anomalous gauge-invariant operators are also presented. Finally, using some illustrative values of anomalous $H W W$ couplings in a more phenomenological parametrization, we indicate the viability of a correlated two-dimensional likelihood analysis to fully exploit the kinematics of the Higgs boson. The latter is particularly relevant to disentangle the SM from CP-violating admixtures. On the whole, we thus conclude that a Higgs factory can considerably improve our understanding of whether the recently discovered scalar is the SM Higgs or not, as evinced from its interactions with a pair of weak gauge bosons.

\section{Acknowledgments}

We thank Taushif Ahmed, Satyanarayan Mukhopadhyay and Narayan Rana for helpful discussions. The work done by SvB was supported by The Claude Leon Foundation. The work of S.B., T.M. and B. Mukhopadhyaya was partially supported by funding available from the Department of Atomic Energy, Government of India for the Regional Centre for Accelerator-based Particle Physics (RECAPP), Harish-Chandra Research Institute. B. Mellado acknowledges the hospitality of RECAPP, Harish-Chandra Research Institute, during the collaboration.

Open Access. This article is distributed under the terms of the Creative Commons Attribution License (CC-BY 4.0), which permits any use, distribution and reproduction in any medium, provided the original author(s) and source are credited. 


\section{References}

[1] ATLAS collaboration, Observation of a new particle in the search for the standard model Higgs boson with the ATLAS detector at the LHC, Phys. Lett. B 716 (2012) 1 [arXiv:1207.7214] [INSPIRE].

[2] CMS collaboration, Observation of a new boson at a mass of $125 \mathrm{GeV}$ with the CMS experiment at the LHC, Phys. Lett. B 716 (2012) 30 [arXiv:1207.7235] [InSPIRE].

[3] S.L. Glashow, Partial-symmetries of weak interactions, Nucl. Phys. 22 (1961) 579 [InSPIRE].

[4] S. Weinberg, A model of leptons, Phys. Rev. Lett. 19 (1967) 1264 [INSPIRE].

[5] N. Svartholm ed. $8^{\text {th }}$ Nobel symposium, C68-05-19, Sweden (1968) [INSPIRE].

[6] F. Englert and R. Brout, Broken symmetry and the mass of gauge vector mesons, Phys. Rev. Lett. 13 (1964) 321 [INSPIRE].

[7] P.W. Higgs, Broken symmetries and the masses of gauge bosons, Phys. Rev. Lett. 13 (1964) 508 [INSPIRE].

[8] P.W. Higgs, Broken symmetries, massless particles and gauge fields, Phys. Lett. 12 (1964) 132 [INSPIRE].

[9] P.W. Higgs, Spontaneous symmetry breakdown without massless bosons, Phys. Rev. 145 (1966) 1156 [INSPIRE].

[10] G.S. Guralnik, C.R. Hagen and T.W.B. Kibble, Global conservation laws and massless particles, Phys. Rev. Lett. 13 (1964) 585 [INSPIRE].

[11] T.W.B. Kibble, Symmetry breaking in non-Abelian gauge theories, Phys. Rev. 155 (1967) 1554 [INSPIRE].

[12] F. Bonnet, M.B. Gavela, T. Ota and W. Winter, Anomalous Higgs couplings at the LHC and their theoretical interpretation, Phys. Rev. D 85 (2012) 035016 [arXiv:1105.5140] [INSPIRE].

[13] J.R. Espinosa, C. Grojean, M. Muhlleitner and M. Trott, Fingerprinting Higgs suspects at the LHC, JHEP 05 (2012) 097 [arXiv: 1202.3697] [INSPIRE].

[14] P.P. Giardino, K. Kannike, M. Raidal and A. Strumia, Reconstructing Higgs boson properties from the LHC and Tevatron data, JHEP 06 (2012) 117 [arXiv: 1203.4254] [INSPIRE].

[15] T. Li, X. Wan, Y.-K. Wang and S.-H. Zhu, Constraints on the universal varying Yukawa couplings: from SM-like to Fermiophobic, JHEP 09 (2012) 086 [arXiv:1203.5083] [INSPIRE].

[16] M. Rauch, Determination of Higgs-boson couplings (SFitter), arXiv:1203.6826 [INSPIRE].

[17] J.R. Espinosa, M. Muhlleitner, C. Grojean and M. Trott, Probing for invisible Higgs decays with global fits, JHEP 09 (2012) 126 [arXiv:1205.6790] [INSPIRE].

[18] J. Ellis and T. You, Global analysis of experimental constraints on a possible Higgs-like particle with mass $125 \mathrm{GeV}$, JHEP 06 (2012) 140 [arXiv: 1204.0464] [INSPIRE].

[19] D. Carmi, A. Falkowski, E. Kuflik and T. Volansky, Interpreting LHC Higgs results from natural new physics perspective, JHEP 07 (2012) 136 [arXiv:1202.3144] [INSPIRE].

[20] M. Dührssen et al., Extracting Higgs boson couplings from CERN LHC data, Phys. Rev. D 70 (2004) 113009 [hep-ph/0406323] [INSPIRE].

[21] R. Lafaye, T. Plehn, M. Rauch, D. Zerwas and M. Dührssen, Measuring the Higgs sector, JHEP 08 (2009) 009 [arXiv:0904.3866] [INSPIRE]. 
[22] N. Desai, D.K. Ghosh and B. Mukhopadhyaya, CP-violating $H W W$ couplings at the Large Hadron Collider, Phys. Rev. D 83 (2011) 113004 [arXiv:1104.3327] [InSPIRE].

[23] M. Klute, R. Lafaye, T. Plehn, M. Rauch and D. Zerwas, Measuring Higgs couplings from LHC data, Phys. Rev. Lett. 109 (2012) 101801 [arXiv:1205.2699] [INSPIRE].

[24] A. Azatov et al., Determining Higgs couplings with a model-independent analysis of $h \rightarrow \gamma \gamma$, JHEP 06 (2012) 134 [arXiv:1204.4817] [INSPIRE].

[25] I. Low, J. Lykken and G. Shaughnessy, Have we observed the Higgs (imposter)?, Phys. Rev. D 86 (2012) 093012 [arXiv: 1207.1093] [INSPIRE].

[26] T. Corbett, O.J.P. Éboli, J. Gonzalez-Fraile and M.C. Gonzalez-Garcia, Constraining anomalous Higgs interactions, Phys. Rev. D 86 (2012) 075013 [arXiv:1207.1344] [INSPIRE].

[27] P.P. Giardino, K. Kannike, M. Raidal and A. Strumia, Is the resonance at $125 \mathrm{GeV}$ the Higgs boson?, Phys. Lett. B 718 (2012) 469 [arXiv:1207.1347] [INSPIRE].

[28] J. Baglio, A. Djouadi and R.M. Godbole, The apparent excess in the Higgs to di-photon rate at the LHC: new physics or QCD uncertainties?, Phys. Lett. B 716 (2012) 203 [arXiv: 1207.1451] [INSPIRE].

[29] J. Ellis and T. You, Global analysis of the Higgs candidate with mass $125 \mathrm{GeV}$, JHEP 09 (2012) 123 [arXiv:1207.1693] [INSPIRE].

[30] M. Montull and F. Riva, Higgs discovery: the beginning or the end of natural EWSB?, JHEP 11 (2012) 018 [arXiv:1207.1716] [INSPIRE].

[31] J.R. Espinosa, C. Grojean, M. Muhlleitner and M. Trott, First glimpses at Higgs' face, JHEP 12 (2012) 045 [arXiv: 1207.1717] [INSPIRE].

[32] D. Carmi, A. Falkowski, E. Kuflik, T. Volansky and J. Zupan, Higgs after the discovery: a status report, JHEP 10 (2012) 196 [arXiv:1207.1718] [INSPIRE].

[33] S. Banerjee, S. Mukhopadhyay and B. Mukhopadhyaya, New Higgs interactions and recent data from the LHC and the Tevatron, JHEP 10 (2012) 062 [arXiv:1207.3588] [INSPIRE].

[34] F. Bonnet, T. Ota, M. Rauch and W. Winter, Interpretation of precision tests in the Higgs sector in terms of physics beyond the standard model, Phys. Rev. D 86 (2012) 093014 [arXiv: 1207.4599] [INSPIRE].

[35] T. Plehn and M. Rauch, Higgs couplings after the discovery, Europhys. Lett. 100 (2012) 11002 [arXiv: 1207.6108] [INSPIRE].

[36] A. Djouadi, Precision Higgs coupling measurements at the LHC through ratios of production cross sections, Eur. Phys. J. C 73 (2013) 2498 [arXiv:1208.3436] [INSPIRE].

[37] B. Batell, S. Gori and L.-T. Wang, Higgs couplings and precision electroweak data, JHEP 01 (2013) 139 [arXiv: 1209.6382] [INSPIRE].

[38] G. Moreau, Constraining extra-fermion(s) from the Higgs boson data, Phys. Rev. D 87 (2013) 015027 [arXiv:1210.3977] [InSPIRE].

[39] G. Bhattacharyya, D. Das and P.B. Pal, Modified Higgs couplings and unitarity violation, Phys. Rev. D 87 (2013) 011702 [arXiv:1212.4651] [InSPIRE].

[40] D. Choudhury, R. Islam and A. Kundu, Anomalous Higgs couplings as a window to new physics, Phys. Rev. D 88 (2013) 013014 [arXiv:1212.4652] [InSPIRE].

[41] G. Bélanger, B. Dumont, U. Ellwanger, J.F. Gunion and S. Kraml, Higgs couplings at the end of 2012, JHEP 02 (2013) 053 [arXiv: 1212.5244] [INSPIRE]. 
[42] M. Klute, R. Lafaye, T. Plehn, M. Rauch and D. Zerwas, Measuring Higgs couplings at a linear collider, Europhys. Lett. 101 (2013) 51001 [arXiv:1301.1322] [INSPIRE].

[43] C. Grojean, E.E. Jenkins, A.V. Manohar and M. Trott, Renormalization group scaling of Higgs operators and $\Gamma(h \rightarrow \gamma \gamma)$, JHEP 04 (2013) 016 [arXiv:1301.2588] [INSPIRE].

[44] K. Cheung, J.S. Lee and P.-Y. Tseng, Higgs precision (Higgcision) era begins, JHEP 05 (2013) 134 [arXiv:1302.3794] [INSPIRE].

[45] J. Elias-Miró, J.R. Espinosa, E. Masso and A. Pomarol, Renormalization of dimension-six operators relevant for the Higgs decays $h \rightarrow \gamma \gamma, \gamma Z$, JHEP 08 (2013) 033 [arXiv:1302.5661] [INSPIRE].

[46] J. Ellis, V. Sanz and T. You, Associated production evidence against Higgs impostors and anomalous couplings, Eur. Phys. J. C 73 (2013) 2507 [arXiv: 1303.0208] [INSPIRE].

[47] P.P. Giardino, K. Kannike, I. Masina, M. Raidal and A. Strumia, The universal Higgs fit, JHEP 05 (2014) 046 [arXiv: 1303.3570] [InSPIRE].

[48] R. Contino, M. Ghezzi, C. Grojean, M. Muhlleitner and M. Spira, Effective Lagrangian for a light Higgs-like scalar, JHEP 07 (2013) 035 [arXiv: 1303.3876] [INSPIRE].

[49] J. Ellis and T. You, Updated global analysis of Higgs couplings, JHEP 06 (2013) 103 [arXiv: 1303.3879] [INSPIRE].

[50] A. Djouadi and G. Moreau, The couplings of the Higgs boson and its CP properties from fits of the signal strengths and their ratios at the $7+8 \mathrm{TeV}$ LHC, Eur. Phys. J. C 73 (2013) 2512 [arXiv: 1303.6591] [INSPIRE].

[51] W.-F. Chang, W.-P. Pan and F. Xu, Effective gauge-Higgs operators analysis of new physics associated with the Higgs boson, Phys. Rev. D 88 (2013) 033004 [arXiv:1303.7035] [INSPIRE].

[52] T. Corbett, O.J.P. Éboli, J. Gonzalez-Fraile and M.C. Gonzalez-Garcia, Determining triple gauge boson couplings from Higgs data, Phys. Rev. Lett. 111 (2013) 011801 [arXiv: 1304.1151] [INSPIRE].

[53] B. Dumont, S. Fichet and G. von Gersdorff, A Bayesian view of the Higgs sector with higher dimensional operators, JHEP 07 (2013) 065 [arXiv: 1304.3369] [INSPIRE].

[54] J. Elias-Miro, J.R. Espinosa, E. Masso and A. Pomarol, Higgs windows to new physics through D $=6$ operators: constraints and one-loop anomalous dimensions, JHEP 11 (2013) 066 [arXiv: 1308.1879] [INSPIRE].

[55] M.B. Einhorn and J. Wudka, Higgs-boson couplings beyond the standard model, Nucl. Phys. B 877 (2013) 792 [arXiv: 1308.2255] [INSPIRE].

[56] A. Pomarol and F. Riva, Towards the ultimate SM fit to close in on Higgs physics, JHEP 01 (2014) 151 [arXiv:1308.2803] [INSPIRE].

[57] ATLAS collaboration, Search for invisible decays of a Higgs boson produced in association with a $Z$ boson in ATLAS, Phys. Rev. Lett. 112 (2014) 201802 [arXiv:1402.3244] [INSPIRE].

[58] CMS collaboration, M.E. Chasco, Search for invisible Higgs boson production with the CMS detector at the LHC, arXiv:1310.1002 [INSPIRE].

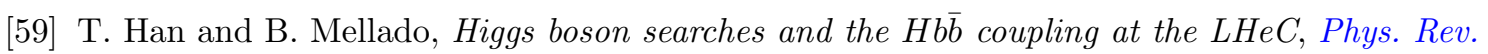
D 82 (2010) 016009 [arXiv:0909.2460] [INSPIRE].

[60] S.S. Biswal, R.M. Godbole, B. Mellado and S. Raychaudhuri, Azimuthal angle probe of anomalous $H W W$ couplings at a high energy ep collider, Phys. Rev. Lett. 109 (2012) 261801 [arXiv: 1203.6285] [INSPIRE]. 
[61] S.S. Biswal, R.M. Godbole, R.K. Singh and D. Choudhury, Signatures of anomalous VVH interactions at a linear collider, Phys. Rev. D 73 (2006) 035001 [Erratum ibid. D 74 (2006) 039904] [hep-ph/0509070] [INSPIRE].

[62] T. Corbett, O.J.P. Éboli, J. Gonzalez-Fraile and M.C. Gonzalez-Garcia, Robust determination of the scalar boson couplings, arXiv:1306.0006 [INSPIRE].

[63] E. Massó and V. Sanz, Limits on anomalous couplings of the Higgs boson to electroweak gauge bosons from LEP and the LHC, Phys. Rev. D 87 (2013) 033001 [arXiv:1211.1320] [INSPIRE].

[64] T. Corbett, O.J.P. Éboli, J. Gonzalez-Fraile and M.C. Gonzalez-Garcia, Robust determination of the Higgs couplings: power to the data, Phys. Rev. D 87 (2013) 015022 [arXiv:1211.4580] [INSPIRE].

[65] A. Falkowski, F. Riva and A. Urbano, Higgs at last, JHEP 11 (2013) 111 [arXiv:1303.1812] [INSPIRE].

[66] S. Banerjee, S. Mukhopadhyay and B. Mukhopadhyaya, Higher dimensional operators and the LHC Higgs data: the role of modified kinematics, Phys. Rev. D 89 (2014) 053010 [arXiv:1308.4860] [INSPIRE].

[67] W. Buchmüller and D. Wyler, Effective Lagrangian analysis of new interactions and flavor conservation, Nucl. Phys. B 268 (1986) 621 [INSPIRE].

[68] B. Grzadkowski, M. Iskrzynski, M. Misiak and J. Rosiek, Dimension-six terms in the standard model Lagrangian, JHEP 10 (2010) 085 [arXiv: 1008.4884] [INSPIRE].

[69] K. Hagiwara, R. Szalapski and D. Zeppenfeld, Anomalous Higgs boson production and decay, Phys. Lett. B 318 (1993) 155 [hep-ph/9308347] [INSPIRE].

[70] M.C. Gonzalez-Garcia, Anomalous Higgs couplings, Int. J. Mod. Phys. A 14 (1999) 3121 [hep-ph/9902321] [INSPIRE].

[71] A. Djouadi, R.M. Godbole, B. Mellado and K. Mohan, Probing the spin-parity of the Higgs boson via jet kinematics in vector boson fusion, Phys. Lett. B 723 (2013) 307 [arXiv:1301.4965] [INSPIRE].

[72] A. Alloul, N.D. Christensen, C. Degrande, C. Duhr and B. Fuks, FeynRules $2.0-a$ complete toolbox for tree-level phenomenology, Comput. Phys. Commun. 185 (2014) 2250 [arXiv: 1310.1921] [INSPIRE].

[73] C. Degrande et al., UFO - the Universal FeynRules Output, Comput. Phys. Commun. 183 (2012) 1201 [arXiv: 1108. 2040] [INSPIRE].

[74] J. Alwall, M. Herquet, F. Maltoni, O. Mattelaer and T. Stelzer, MadGraph 5: going beyond, JHEP 06 (2011) 128 [arXiv: 1106.0522] [INSPIRE].

[75] J.A.M. Vermaseren, New features of FORM, math-ph/0010025 [INSPIRE].

[76] S. Heinemeyer et al., Toward high precision Higgs-boson measurements at the international linear $e^{+} e^{-}$collider, hep-ph/0511332 [INSPIRE].

[77] G. Altarelli, B. Mele and F. Pitolli, Heavy Higgs production at future colliders, Nucl. Phys. B 287 (1987) 205 [INSPIRE].

[78] A. Pomarol, D. Zeppenfeld and M. Perelstein, private communications.

[79] K. Hagiwara, R.D. Peccei, D. Zeppenfeld and K. Hikasa, Probing the weak boson sector in $e^{+} e^{-} \rightarrow W^{+} W^{-}$, Nucl. Phys. B 282 (1987) 253 [InSPIRE]. 